\title{
MOJAVE. X. PARSEC-SCALE JET ORIENTATION VARIATIONS AND SUPERLUMINAL MOTION IN ACTIVE GALACTIC NUCLEI
}

\author{
M. L. Lister ${ }^{1}$, M. F. Aller ${ }^{2}$, H. D. Aller ${ }^{2}$, D. C. Homan ${ }^{3}$, K. I. Kellermann ${ }^{4}$, Y. Y. Kovalev ${ }^{5,6}$,

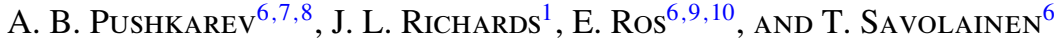 \\ ${ }^{1}$ Department of Physics, Purdue University, 525 Northwestern Avenue, West Lafayette, IN 47907, USA; mlister@ purdue.edu \\ 2 Department of Astronomy, University of Michigan, 817 Dennison Building, Ann Arbor, MI 48109, USA \\ ${ }^{3}$ Department of Physics, Denison University, Granville, OH 43023, USA \\ ${ }^{4}$ National Radio Astronomy Observatory, 520 Edgemont Road, Charlottesville, VA 22903, USA \\ ${ }^{5}$ Astro Space Center of Lebedev Physical Institute, Profsoyuznaya 84/32, 117997 Moscow, Russia \\ ${ }^{6}$ Max-Planck-Institut für Radioastronomie, Auf dem Hügel 69, D-53121 Bonn, Germany \\ ${ }^{7}$ Pulkovo Observatory, Pulkovskoe Chaussee 65/1, 196140 St. Petersburg, Russia \\ ${ }^{8}$ Crimean Astrophysical Observatory, 98409 Nauchny, Crimea, Ukraine \\ ${ }^{9}$ Observatori Astronòmic, Universitat de València, Parc Científic, C. Catedrático José Beltrán 2, E-46980 Paterna, València, Spain \\ ${ }^{10}$ Departament d'Astronomia i Astrofísica, Universitat de València, C. Dr. Moliner 50, E-46100 Burjassot, València, Spain \\ Received 2013 June 6; accepted 2013 August 5; published 2013 October 8
}

\begin{abstract}
We describe the parsec-scale kinematics of 200 active galactic nucleus (AGN) jets based on 15 GHz Very Long Baseline Array (VLBA) data obtained between 1994 August 31 and 2011 May 1. We present new VLBA 15 GHz images of these and 59 additional AGNs from the MOJAVE and $2 \mathrm{~cm}$ Survey programs. Nearly all of the 60 most heavily observed jets show significant changes in their innermost position angle over a 12-16 yr interval, ranging from $10^{\circ}$ to $150^{\circ}$ on the sky, corresponding to intrinsic variations of $\sim 0.5$ to $\sim 2^{\circ}$. The BL Lac jets show smaller variations than quasars. Roughly half of the heavily observed jets show systematic position angle trends with time, and 20 show indications of oscillatory behavior. The time spans of the data sets are too short compared to the fitted periods (5-12 yr), however, to reliably establish periodicity. The rapid changes and large jumps in position angle seen in many cases suggest that the superluminal AGN jet features occupy only a portion of the entire jet cross section and may be energized portions of thin instability structures within the jet. We have derived vector proper motions for 887 moving features in 200 jets having at least five VLBA epochs. For 557 well-sampled features, there are sufficient data to additionally study possible accelerations. We find that the moving features are generally non-ballistic, with $70 \%$ of the well-sampled features showing either significant accelerations or non-radial motions.

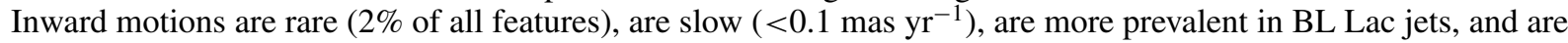
typically found within 1 mas of the unresolved core feature. There is a general trend of increasing apparent speed with distance down the jet for both radio galaxies and BL Lac objects. In most jets, the speeds of the features cluster around a characteristic value, yet there is a considerable dispersion in the distribution. Orientation variations within the jet cannot fully account for the dispersion, implying that the features have a range of Lorentz factor and/or pattern speed. Very slow pattern speed features are rare, comprising only $4 \%$ of the sample, and are more prevalent in radio galaxy and BL Lac jets. We confirm a previously reported upper envelope to the distribution of speed versus beamed luminosity for moving jet features. Below $10^{26} \mathrm{~W} \mathrm{~Hz}^{-1}$ there is a fall-off in maximum speed with decreasing $15 \mathrm{GHz}$ radio luminosity. The general shape of the envelope implies that the most intrinsically powerful AGN jets have a wide range of Lorentz factors up to $\sim 40$, while intrinsically weak jets are only mildly relativistic.
\end{abstract}

Key words: BL Lacertae objects: general - galaxies: active - galaxies: jets - quasars: general - radio continuum: galaxies

Online-only material: color figures, figure sets, machine-readable and VO tables

\section{INTRODUCTION}

High-resolution multi-epoch radio observations of jetted outflows associated with active galactic nuclei (AGNs) have contributed substantially to our understanding of the immediate environments of supermassive black holes, by providing direct measurements of jet flow kinematics and magnetic field properties. Since its construction in 1994, the Very Long Baseline Array (VLBA) has been used to regularly image the brightest radio-loud AGN jets and study their evolution on parsec-scales (Kellermann et al. 2004). The VLBA $2 \mathrm{~cm}$ Survey (Kellermann et al. 1998) sampled the jet kinematics of 110 AGNs and was succeeded in 2002 by the MOJAVE program, which added full polarization imaging and defined a complete northern-sky radio flux-density-limited sample (Lister \&
Homan 2005, hereafter Paper I). Kinematic results for 127 MOJAVE jets based on data spanning 1994-2007 were presented by Lister et al. (2009b, hereafter Paper VI) and Homan et al. (2009, hereafter Paper VII). They showed that bright jet features typically exhibit apparent superluminal speeds and accelerated motions. These findings are consistent with the widely accepted picture of high bulk Lorentz factor jets viewed at angles very close to the line of sight, i.e., blazars. Although blazars are quite rare in the general AGN parent population, their predominance in the flux-density-limited MOJAVE sample is a direct result of Doppler orientation bias (Orr \& Browne 1982), since the observed flux densities of aligned, fast jets are highly Doppler boosted by relativistic aberration effects.

The MOJAVE program has confirmed an important trend, first reported by Vermeulen (1995) in the Caltech-Jodrell AGN 
Table 1

General Properties of AGNs in the Combined Samples

\begin{tabular}{|c|c|c|c|c|c|c|}
\hline $\begin{array}{l}\text { B1950 } \\
\text { (1) }\end{array}$ & $\begin{array}{l}\text { Alias } \\
(2)\end{array}$ & $\begin{array}{l}\text { 2FGL Assoc. } \\
\text { (3) }\end{array}$ & $\begin{array}{c}z \\
(4)\end{array}$ & $\begin{array}{l}\text { Ref. } \\
(5)\end{array}$ & $\begin{array}{l}\text { Opt. } \\
(6)\end{array}$ & $\begin{array}{c}\text { Sample } \\
(7)\end{array}$ \\
\hline $0003+380$ & S4 0003+38 & J0006.1+3821 & 0.229 & Schramm et al. (1994) & Q & $\mathrm{L}$ \\
\hline $0003-066$ & NRAO 005 & $\ldots$ & 0.3467 & Jones et al. (2005) & $\mathrm{B}$ & $\mathrm{R}$ \\
\hline $0007+106$ & III Zw 2 & $\ldots$ & 0.0893 & Sargent (1970) & $\mathrm{G}$ & $\mathrm{R}, \mathrm{L}$ \\
\hline $0010+405$ & $4 \mathrm{C}+40.01$ & $\ldots$ & 0.256 & Thompson et al. (1992) & Q & $\mathrm{L}$ \\
\hline $0015-054$ & PMN J0017-0512 & J0017.6-0510 & 0.226 & Shaw et al. (2012) & Q & $\mathrm{G}, \mathrm{L}$ \\
\hline $0016+731$ & S5 0016+73 & $\ldots$ & 1.781 & Lawrence et al. (1986) & Q & $\mathrm{R}$ \\
\hline 0048-097 & PKS 0048-09 & J0050.6-0929 & 0.635 & Landoni et al. (2012) & $\mathrm{B}$ & $\mathrm{G}, \mathrm{R}$ \\
\hline $0055+300$ & NGC 315 & $\ldots$ & 0.0165 & Huchra et al. (1999) & $\mathrm{G}$ & $\mathrm{L}$ \\
\hline $0059+581$ & TXS 0059+581 & J0102.7+5827 & 0.644 & Sowards-Emmerd et al. (2005) & Q & $\mathrm{R}$ \\
\hline $0106+013$ & $4 \mathrm{C}+01.02$ & J0108.6+0135 & 2.099 & Hewett et al. (1995) & Q & $\mathrm{G}, \mathrm{R}$ \\
\hline $0109+224$ & S2 $0109+22$ & $\mathrm{~J} 0112.1+2245$ & 0.265 & Shaw et al. (2012) & $\mathrm{B}$ & $\mathrm{G}, \mathrm{R}$ \\
\hline $0109+351$ & B2 $0109+35$ & $\ldots$ & 0.450 & Hook et al. (1996) & Q & $\mathrm{R}$ \\
\hline $0110+318$ & $4 C+31.03$ & J0112.8+3208 & 0.603 & Wills \& Wills (1976) & Q & $\mathrm{G}$ \\
\hline $0111+021$ & UGC 00773 & $\ldots$ & 0.047 & Wills \& Wills (1976) & $\mathrm{B}$ & $\mathrm{L}$ \\
\hline $0116-219$ & OC -228 & J0118.8-2142 & 1.165 & Wright et al. (1983) & Q & $\mathrm{G}$ \\
\hline
\end{tabular}

Notes. Columns are as follows: (1) B1950 name, (2) other name, (3) 2FGL catalog name, (4) redshift, (5) literature reference for redshift and optical classification, (6) optical classification, where B = BL Lac, Q = quasar, G = radio galaxy, $\mathrm{N}=$ narrow-line Seyfert 1, and U = unidentified, (7) sample membership, where $\mathrm{G}=1 \mathrm{FM} \gamma$-ray-selected sample, $\mathrm{R}=$ MOJAVE 1.5 Jy sample, and L = low-luminosity sample.

${ }^{\text {a }}$ Known TeV emitter (http://tevcat.uchicago.edu).

(This table is available in its entirety in machine-readable and Virtual Observatory (VO) forms in the online journal. A portion is shown here for guidance regarding its form and content.)

survey, in which jets with the fastest superluminal speeds all tend to have high Doppler boosted radio luminosities. To first order, such a trend might be expected from orientation and Doppler boosting effects, but an analysis by Cohen et al. (2007) and Monte Carlo simulations presented in Paper VI indicated that there is a correlation between intrinsic jet speed and intrinsic (debeamed) luminosity present in the population. In the absence of such a correlation, we would expect to see highly superluminal jets at much lower boosted radio luminosities.

In order to further investigate these issues, we expanded the MOJAVE program in 2006 to include regular VLBA imaging of additional low-luminosity AGN jets. In 2009, we expanded the sample again to encompass new $\gamma$-ray loud blazar jets detected by Fermi (Lister et al. 2011).

We present new VLBA $15 \mathrm{GHz}$ images of the original 135 source MOJAVE flux-density-limited sample obtained between 2007 September 6 and 2011 May 1. We also present VLBA images of 124 additional AGNs from three new AGN jet samples, based on $15 \mathrm{GHz}$ VLBA data obtained between 1994 August 31 and 2011 May 1 from the NRAO archive and the MOJAVE program (Lister et al. 2009a, hereafter Paper V). These include a complete radio-selected sample above $1.5 \mathrm{Jy}$, a complete $\gamma$-ray-selected sample, and a representative low-luminosity AGN jet sample. We use these data to present an updated kinematics analysis of the 135 jets in the original MOJAVE flux-density-limited sample and first ever kinematics analyses of 65 jets for which we have obtained at least five VLBA epochs.

The overall layout of the paper is as follows. In Sections 2 and 3 we discuss the samples and observational data, respectively. In Section 4 we describe our method of modeling the individual jet features and their kinematic properties. We discuss overall trends in the data in Section 5 and summarize our findings in Section 6. We adopt a cosmology with $\Omega_{m}=0.27$, $\Omega_{\Lambda}=0.73$, and $H_{0}=71 \mathrm{~km} \mathrm{~s}^{-1} \mathrm{Mpc}^{-1}$. We refer to the radio sources throughout using either B1950 nomenclature or commonly used aliases, which we list in Table 1.

\section{AGN SAMPLE DEFINITIONS}

\subsection{Radio-selected MOJAVE 1.5 Jy Sample}

Unlike blazar surveys in the optical or soft X-ray regimes, the radio emission from the brightest radio-loud blazars is not substantially obscured by or blended with emission from the host galaxy. A VLBA-selected sample thus provides a very "clean" blazar sample, namely, one selected on the basis of (beamed synchrotron) jet emission.

In Paper V we described the original radio-selected MOJAVE sample of 135 AGNs, which was based on the $15 \mathrm{GHz}$ VLBA flux density exceeding $1.5 \mathrm{Jy}\left(2 \mathrm{Jy}\right.$ for declination $<0^{\circ}$ ) at any epoch during the period 1994.0-2004.0. The sky region was limited to declination $\geqslant-20^{\circ}$ and the Galactic plane region $|b|<10^{\circ}$. In order to encompass a broader range of Fermi-detected AGNs, particularly those that recently entered an active state, we updated our radio-selection criteria in 2011 to form the complete MOJAVE $1.5 \mathrm{Jy}$ sample. The latter now consists of all known non-gravitationally lensed AGNs with $\mathrm{J} 2000$ declination $>-30^{\circ}$ (no Galactic plane restriction) and VLBA flux density $S_{15 \mathrm{GHz}}>1.5 \mathrm{Jy}$ at any epoch between 1994.0 and 2010.0. The new list results in a larger overlap with the Fermi AGN catalog (Ackermann et al. 2011) and simplifies the determination of luminosity functions (e.g., Cara $\&$ Lister 2008), which are useful for studies of the extragalactic background light and blazar parent populations.

The overall properties of the sample are summarized in Table 1, where the "R" notation in Column (7) indicates $1.5 \mathrm{Jy}$ radio sample membership. There are 183 AGNs in total (see Table 2), with the sample being heavily dominated by flat-spectrum radio quasars $(78 \%)$ and BL Lac objects $(16 \%)$. The optical classifications are $98 \%$ complete, with redshifts available for $96 \%$ of the sample.

\section{2. $\gamma$-ray-selected IFM Sample}

The continuous all-sky coverage of the LAT instrument on board the Fermi satellite has significantly improved our 
Table 2

Optical Classification Summary of AGN Samples

\begin{tabular}{lccc}
\hline \hline & 1.5 Jy Radio & 1FM $\gamma$-ray & Low-luminosity \\
\hline Quasars & 142 & 72 & 7 \\
BL Lac objects & 29 & 42 & 20 \\
Radio galaxies & 8 & 1 & 16 \\
Narrow-line Seyfert 1s & 0 & 1 & 0 \\
Unidentified & 4 & 0 & 0 \\
\hline Total & 183 & 116 & 43 \\
\hline
\end{tabular}

Note. Some AGNs belong to two or more of the samples listed above (see Figure 1).

knowledge of the blazar population at $\gamma$-ray energies above $100 \mathrm{MeV}$ by identifying nearly 1000 AGNs associated with $\gamma$-ray sources (Ackermann et al. 2011). For the joint LAT team-MOJAVE study of Lister et al. (2011), we constructed a $\gamma$-ray sample based on the initial 11 month First Fermi AGN catalog (Abdo et al. 2010). The specific criteria were: (1) average integrated $>0.1 \mathrm{GeV}$ energy flux $\geqslant 3 \times 10^{-11} \mathrm{erg} \mathrm{cm}^{-2} \mathrm{~s}^{-2}$ between 2008 August 4 and 2009 July 5, (2) J2000 declination $>30^{\circ}$, (3) galactic latitude $|b|>10^{\circ}$, and (4) source not associated with a gravitational lens. The sample is complete with respect to $\gamma$-ray flux, with the exception of two $\gamma$-ray sources (1FGL J1653.6-0158 and 1FGL J2339.7-0531) that were dropped since they had no unambiguous radio counterpart.

The overall properties of the sample are described by Lister et al. (2011) and summarized in Table 1, where the " $G$ " notation in Column (7) indicates $\gamma$-ray sample membership. There are 116 AGNs in total (Table 2), 56 of which are in common with the MOJAVE 1.5 Jy sample. Like our radio-selected sample, it is heavily dominated by blazars, but contains a larger fraction of BL Lac objects (36\%). The remainder of the sample are quasars, with the exception of the nearby radio galaxy NGC 1275 (3C 84) and the narrow-line Seyfert 1 galaxy PMN J0948+0022.

\subsection{Low-luminosity Compact AGN Sample}

In 2006 we expanded the MOJAVE program to include regular VLBA observations of 16 AGNs with VLBA $15 \mathrm{GHz}$ luminosities below $<10^{26} \mathrm{~W} \mathrm{~Hz}^{-1}$. These were chosen from the VLBA Calibrator Survey (Beasley et al. 2002; Fomalont et al. 2003; Petrov et al. 2005, 2006; Kovalev et al. 2007; Petrov et al. 2008), based on the following criteria: (1) $8 \mathrm{GHz}$ VLBA flux density greater than $0.35 \mathrm{Jy},(2) z \leqslant 0.3$, and (3) $\mathrm{J} 2000$ declination $>-30^{\circ}$. By adding the AGN already in the MOJAVE program that met these criteria, we obtained a final sample of 43 low-luminosity compact AGNs, as indicated by the "L" notation in Column (7) of Table 1. Although the latter would not typically be considered as low-luminosity among the general radio-loud AGN population, we will refer to them as the "low-luminosity" sample, in comparison with the typically high-luminosity blazars in our radio- and $\gamma$-ray-selected samples. Due to the lack of redshift information for the full VLBA Calibrator Survey, our low-luminosity sample is not complete. However, it is a useful representative set for examining the kinematics of weaker jets and in this respect complements other small-sample low-luminosity AGN very long baseline interferometry (VLBI) monitoring programs (e.g., Giovannini et al. 2001; Piner et al. 2010).

These three samples, comprising 259 AGNs in total, provide a broad cross section of AGN types among bright, compact radio sources. The overlap among the samples is shown in Figure 1.

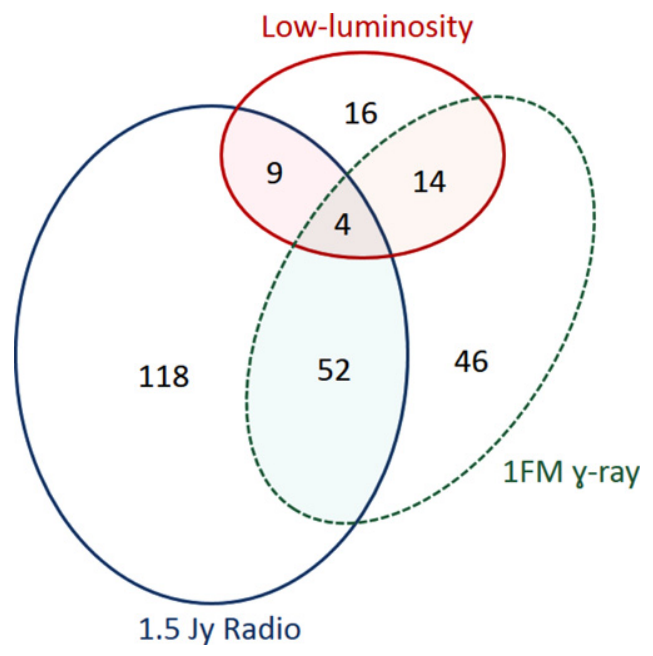

Figure 1. Area-proportional Venn diagram with labels indicating the total number of AGNs in each subset of the MOJAVE 1.5 Jy, 1FM $\gamma$-ray-selected, and low-luminosity AGN samples.

(A color version of this figure is available in the online journal.)

Since only 200 of these sources had at least five VLBA epochs as of 2011 May, the kinematic data are incomplete. In particular, the AGNs with missing data tend to be among the weaker radioand $\gamma$-ray-selected AGNs that were added later in the MOJAVE survey. A statistical inter-comparison of these samples will be presented in future papers in this series once a full, unbiased data set has been collected.

\section{VLBA OBSERVATIONS AND DATA REDUCTION}

In Paper $\mathrm{V}$, we presented $15 \mathrm{GHz}$ VLBA images of the 135 AGNs in the original MOJAVE flux-density-limited sample based on data from the MOJAVE programs spanning 1994 August 31 to 2007 September 9, the VLBA 2m Survey, and the NRAO archive. ${ }^{11}$ In Figure 2 we show naturally weighted $15 \mathrm{GHz}$ VLBA images derived from newly acquired VLBA data on these AGNs up to 2011 May 1, as well as VLBA data from 1994 August 31 to 2011 May 1 on 124 AGNs in our new samples.

The multi-epoch observations for each AGN, along with the corresponding image parameters, are listed in Table 3. Column 3 gives the VLBA project code for each observation, along with an indicator as to whether it is from the MOJAVE program, the VLBA $2 \mathrm{~cm}$ Survey, or the NRAO archive. For the latter, we considered only archival data with at least four scans spanning a range of hour angle, and which included eight or more VLBA antennas. The VLBA $2 \mathrm{~cm}$ Survey observations (1994-2002) analyzed by Kellermann et al. (2004) consist of approximately $1 \mathrm{hr}$ integrations on each AGN, broken up into approximately 6-8 minute scans separated in hour angle to improve the interferometric coverage. A similar observing method and integration times were used in the full polarization MOJAVE observations from 2002 May to 2007 September (VLBA codes BL111, BL123, BL137, and BL149; see Table 3) and are described in Paper I. During 2006 (VLBA code BL137), the $15 \mathrm{GHz}$ integration times were shortened by a factor of $\sim 3$ to accommodate interleaved scans at three other observing frequencies $(8.1,8.4$, and $12.1 \mathrm{GHz})$. The latter data were presented by Hovatta et al. (2012) and Pushkarev et al. (2012). The MOJAVE and $2 \mathrm{~cm}$ Survey observations were recorded at

\footnotetext{
11 http://archive.nrao.edu/
} 

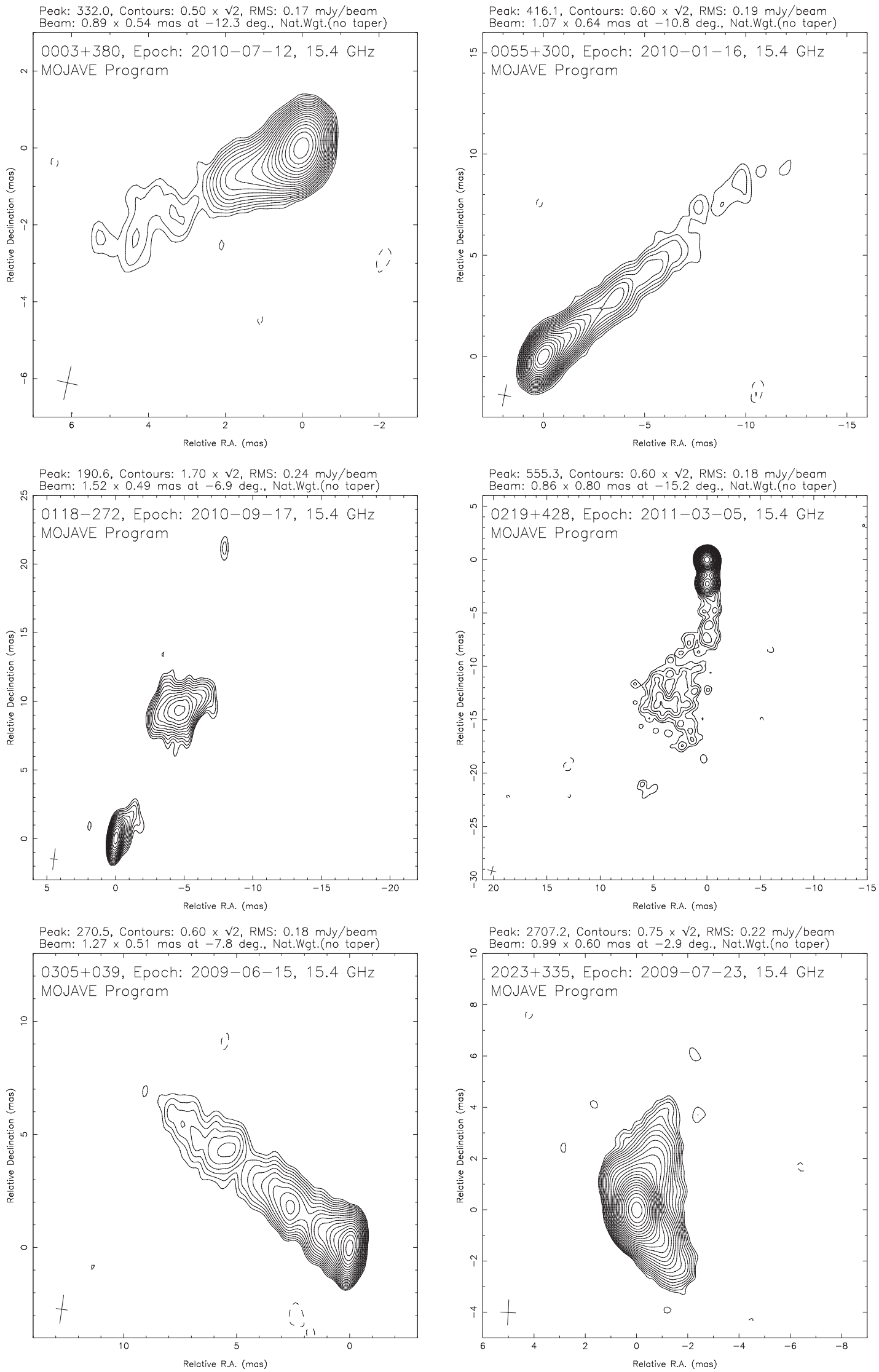

Figure 2. Naturally weighted $15 \mathrm{GHz}$ total intensity VLBA contour images of individual epoch observations of the MOJAVE AGN sample. The contours are in successive powers of $\sqrt{2}$ times the base contour level, as listed in Table 3 and at the top of each panel. Because of self-calibration, in some cases the origin may be coincident with the brightest feature in the image, rather than the putative core feature listed in Table 4.

(The complete figure set (1753 images) is available in the online journal.) 
Table 3

Summary of $15 \mathrm{GHz}$ Image Parameters

\begin{tabular}{|c|c|c|c|c|c|c|c|c|c|c|}
\hline $\begin{array}{l}\text { Source } \\
\text { (1) }\end{array}$ & $\begin{array}{c}\text { Epoch } \\
\text { (2) }\end{array}$ & $\begin{array}{l}\text { VLBA } \\
\text { Code } \\
(3)\end{array}$ & $\begin{array}{c}\text { Freq. } \\
(\mathrm{GHz}) \\
(4)\end{array}$ & $\begin{array}{c}B_{\text {maj }} \\
(\text { mas) } \\
(5)\end{array}$ & $\begin{array}{c}B_{\min } \\
(\mathrm{mas}) \\
(6)\end{array}$ & $\begin{array}{l}B_{\mathrm{pa}} \\
\left({ }^{\circ}\right) \\
(7)\end{array}$ & $\begin{array}{c}I_{\text {tot }} \\
(\mathrm{Jy}) \\
(8)\end{array}$ & $\begin{array}{c}\mathrm{rms} \\
\left(\mathrm{mJy} \mathrm{bm}^{-1}\right) \\
(9)\end{array}$ & $\begin{array}{c}I_{\text {base }} \\
\left(\mathrm{mJy} \mathrm{bm}^{-1}\right) \\
(10)\end{array}$ & $\begin{array}{l}\text { Fig. } \\
\text { Num. }\end{array}$ \\
\hline \multirow[t]{8}{*}{$0003+380$} & 2006 Mar 9 & $\mathrm{BL}_{137 \mathrm{~B}^{\mathrm{a}}}$ & 15.4 & 1.01 & 0.73 & 18 & 0.649 & 0.4 & 1.3 & 2.1 \\
\hline & 2006 Dec 1 & BL137L ${ }^{a}$ & 15.4 & 0.85 & 0.58 & -17 & 0.511 & 0.4 & 1.2 & 2.2 \\
\hline & 2007 Mar 28 & BL137Pa & 15.4 & 0.86 & 0.61 & -15 & 0.602 & 0.3 & 1.0 & 2.3 \\
\hline & 2007 Aug 24 & BL149AM $^{\mathrm{a}}$ & 15.4 & 0.92 & 0.58 & -28 & 0.554 & 0.3 & 0.8 & 2.4 \\
\hline & 2008 May 1 & $\mathrm{BL}_{149 \mathrm{AO}^{\mathrm{a}}}$ & 15.4 & 0.82 & 0.57 & -9 & 0.806 & 0.2 & 0.7 & 2.5 \\
\hline & $2008 \mathrm{Jul} 17$ & $\mathrm{BL}_{149 \mathrm{AK}^{\mathrm{a}}}$ & 15.4 & 0.84 & 0.55 & -12 & 0.725 & 0.2 & 0.6 & 2.6 \\
\hline & 2009 Mar 25 & $\mathrm{BL}^{149 \mathrm{BJ}^{\mathrm{a}}}$ & 15.4 & 0.84 & 0.62 & -12 & 0.435 & 0.2 & 0.5 & 2.7 \\
\hline & $2010 \mathrm{Jul} 12$ & $\mathrm{BL}_{149 \mathrm{CL}^{\mathrm{a}}}$ & 15.4 & 0.89 & 0.54 & -12 & 0.438 & 0.2 & 0.5 & 2.8 \\
\hline \multirow[t]{5}{*}{$0003-066$} & $2008 \mathrm{Jul} 30$ & $\mathrm{BL}_{149 \mathrm{AL}^{\mathrm{a}}}$ & 15.4 & 1.38 & 0.53 & -8 & 1.951 & 0.2 & 0.7 & 2.9 \\
\hline & 2009 May 2 & $\mathrm{BL}_{149 \mathrm{BK}^{\mathrm{a}}}$ & 15.4 & 1.21 & 0.48 & -7 & 2.513 & 0.2 & 0.6 & 2.10 \\
\hline & 2009 Oct 27 & $\mathrm{BL}^{149 \mathrm{CC}^{\mathrm{a}}}$ & 15.4 & 1.71 & 0.56 & -13 & 2.141 & 0.2 & 1.0 & 2.11 \\
\hline & 2010 Aug 6 & $\mathrm{BL}^{149 C \mathrm{CM}^{\mathrm{a}}}$ & 15.4 & 1.33 & 0.59 & 1 & 2.143 & 0.2 & 0.6 & 2.12 \\
\hline & 2010 Nov 29 & $\mathrm{BL}^{149 C \mathrm{Y}^{\mathrm{a}}}$ & 15.4 & 1.48 & 0.53 & -8 & 2.055 & 0.2 & 0.5 & 2.13 \\
\hline $0007+106$ & 2008 Aug 25 & $\mathrm{BL}_{149 \mathrm{BB}^{\mathrm{a}}}$ & 15.4 & 1.17 & 0.49 & -11 & 0.511 & 0.3 & 0.8 & 2.14 \\
\hline
\end{tabular}

Notes. Columns are as follows: (1) B1950 name, (2) date of VLBA observation, (3) VLBA experiment code, (4) observing frequency (GHz), (5) FWHM major axis of restoring beam (milliarcseconds), (6) FWHM minor axis of restoring beam (milliarcseconds), (7) position angle of major axis of restoring beam (degrees), (8) total $I$ flux density (Jy), (9) rms noise level of image (mJy per beam), (10) lowest $I$ contour (mJy per beam), and (11) figure number.

a Full polarization MOJAVE epoch.

b $2 \mathrm{~cm}$ VLBA Survey epoch.

(This table is available in its entirety in machine-readable and Virtual Observatory (VO) forms in the online journal. A portion is shown here for guidance regarding its form and content.)

a data rate of $128 \mathrm{Mbps}$, which was increased to $256 \mathrm{Mbps}$ in the epochs from 2007 July 3 to 2008 September 12 inclusive, and 512 Mbps thereafter. Beginning with the 2007 January 6 epoch, we increased the number of AGNs observed in each $24 \mathrm{hr}$ MOJAVE session from 18 to 25 AGNs to accommodate our expanded monitoring sample described in Section 2. On 2009 February 25 we increased this further to 30 AGNs per session.

\section{DATA ANALYSIS}

\subsection{Gaussian Model Fitting}

As in Paper VI, we modeled the $(u, v)$ visibility data at all AGN epochs using a series of Gaussian components in the Difmap software package (Shepherd 1997). In the majority of cases, we used circular Gaussians for jet features and occasionally (when necessary) elliptical Gaussians for the core feature. The latter was typically the brightest feature at the extreme end of a one-sided jet in most sources (see the Appendix and Paper VI for a discussion of core identifications and two-sided jets in the sample). The parameters of the Gaussian fits are listed in Table 4. In some instances, it was not possible to robustly cross-identify the same components in a jet from one epoch to the next. Those components with robust crossidentifications over at least five epochs for the purpose of kinematics analysis are indicated in Column 10 of Table 4. For the non-robust components, we note that the assignment of the same identification number across epochs in Table 4 does not necessarily indicate a reliable cross-identification.

We estimate errors on the component sizes to be roughly twice the positional error, according to Fomalont (1999). The errors on the peak flux density values are approximately $5 \%$ (see Appendix A of Homan et al. 2002). Based on our previous analysis from Paper VI, we estimate the typical uncertainties in the Gaussian centroid positions to be $\sim 1 / 5$ of the FWHM beam dimensions. For isolated bright and compact components the positional errors are smaller by approximately a factor of 2 . A more quantitative estimate for individual components can be obtained using the scatter of our kinematic fit residuals (Columns 14 and 15 of Table 5). These residuals represent only estimates of the uncertainty of the fits and are likely underestimates in some cases due to possible errors in component cross-identification and/or a low number of epochs. Small variations in the apparent core position, due to changes in opacity and/or newly emerging features, can also contribute to the positional errors. Deviations from linear or simple accelerated motion can also increase the magnitude of the fit residuals (see Section 5.5).

In Paper VI there were eight jets that had no robust jet components. After our analysis using the new data, six remained in this category: $0235+164,0727-115,1124-186$, $1324+224,1739+522$, and $1741-038$. In the case of $0109+224$ and $0742+103$, we did not consider any components to be robust in Paper VI, due to gaps in temporal coverage. We have subsequently obtained several closely spaced VLBA epochs and now consider several slow-moving components in these two jets to be robust.

In Paper VI we listed 0048-097 and 1958-179 as having one robust component each. However, after re-examining the original model fits along with the new data, we have determined that these two jets are too compact at $15 \mathrm{GHz}$ to classify any of their components as robust. Of the new AGNs not in Paper VI, there are six with no robust components: 0716+332, 0946+006, 1921-293, 1959+650, 2023+335, and 2247-283.

\subsection{Jet Kinematics Analysis}

We performed two sets of kinematics analyses on the robust Gaussian jet components in our sample. The first assumed a simple non-accelerating, two-dimensional vector fit to the component position over time, referenced to the core component (which we presumed to be stationary). For the components that had measurements at 10 or more epochs, we also performed a constant acceleration fit (as described in 
Table 4

Fitted Jet Components

\begin{tabular}{|c|c|c|c|c|c|c|c|c|c|}
\hline $\begin{array}{l}\text { Source } \\
\text { (1) }\end{array}$ & $\begin{array}{l}\text { ID } \\
\text { (2) }\end{array}$ & $\begin{array}{c}\text { Epoch } \\
\text { (3) }\end{array}$ & $\begin{array}{c}I \\
(\mathrm{Jy}) \\
(4)\end{array}$ & $\begin{array}{c}r \\
(\mathrm{mas}) \\
(5)\end{array}$ & $\begin{array}{l}\text { P.A. } \\
\left({ }^{\circ}\right) \\
(6)\end{array}$ & $\begin{array}{c}\text { Maj. } \\
\text { (mas) } \\
(7)\end{array}$ & $\begin{array}{c}\text { Ratio } \\
(8)\end{array}$ & $\begin{array}{c}\text { Maj. P.A. } \\
\left({ }^{\circ}\right) \\
(9)\end{array}$ & $\begin{array}{c}\text { Robust? } \\
\text { (10) }\end{array}$ \\
\hline \multirow[t]{15}{*}{$0003+380$} & 0 & 2006 Mar 9 & 0.489 & 0.04 & 290.7 & 0.23 & 0.33 & 292 & $\bar{Y}$ \\
\hline & 1 & & 0.007 & 3.98 & 121.8 & 0.72 & 1.00 & $\ldots$ & $\mathrm{Y}$ \\
\hline & 2 & & 0.042 & 1.25 & 110.5 & 0.51 & 1.00 & $\ldots$ & $\mathrm{Y}$ \\
\hline & 5 & & 0.104 & 0.28 & 114.6 & 0.27 & 1.00 & $\ldots$ & $\mathrm{Y}$ \\
\hline & 6 & & 0.003 & 2.31 & 119.3 & & . & $\ldots$ & $\mathrm{N}$ \\
\hline & 0 & 2006 Dec 1 & 0.320 & 0.10 & 308.1 & 0.25 & 0.29 & 295 & Y \\
\hline & 1 & & 0.005 & 3.65 & 120.8 & 1.63 & 1.00 & $\ldots$ & $\mathrm{Y}$ \\
\hline & 2 & & 0.021 & 1.56 & 111.0 & 0.25 & 1.00 & $\ldots$ & $\mathrm{Y}$ \\
\hline & 4 & & 0.023 & 0.75 & 116.2 & 0.32 & 1.00 & $\ldots$ & $\mathrm{Y}$ \\
\hline & 5 & & 0.145 & 0.45 & 116.3 & 0.05 & 1.00 & $\ldots$ & Y \\
\hline & 0 & 2007 Mar 28 & 0.386 & 0.04 & 309.3 & 0.16 & 0.21 & 307 & Y \\
\hline & 1 & & 0.004 & 4.10 & 119.5 & 0.45 & 1.00 & $\ldots$ & $\mathrm{Y}$ \\
\hline & 2 & & 0.024 & 1.68 & 111.6 & 0.38 & 1.00 & $\ldots$ & Y \\
\hline & 4 & & 0.053 & 0.72 & 117.1 & 0.15 & 1.00 & $\ldots$ & $\mathrm{Y}$ \\
\hline & 5 & & 0.130 & 0.50 & 115.6 & 0.08 & 1.00 & $\ldots$ & $\mathrm{Y}$ \\
\hline
\end{tabular}

Notes. Columns are as follows: (1) B1950 name, (2) component identification number (zero indicates core component), (3) observation epoch, (4) flux density in Jy, (5) position offset from the core component (or map center for the core component entries) in milliarcseconds, (6) position angle with respect to the core component (or map center for the core component entries) in degrees, (7) FWHM major axis of fitted Gaussian in milliarcseconds, (8) axial ratio of fitted Gaussian, (9) major-axis position angle of fitted Gaussian in degrees, and (10) robust component flag.

${ }^{a}$ Individual component epoch not used in kinematic fits.

(This table is available in its entirety in machine-readable and Virtual Observatory (VO) forms in the online journal. A portion is shown here for guidance regarding its form and content.)

Table 5

Kinematic Fit Properties of Jet Components

\begin{tabular}{|c|c|c|c|c|c|c|c|c|c|c|c|c|c|c|}
\hline $\begin{array}{l}\text { Source } \\
\text { (1) }\end{array}$ & $\begin{array}{l}\text { ID } \\
\text { (2) }\end{array}$ & $\begin{array}{l}N \\
\text { (3) }\end{array}$ & $\begin{array}{c}\langle S\rangle \\
(\mathrm{mJy}) \\
(4)\end{array}$ & $\begin{array}{c}\langle R\rangle \\
(\mathrm{mas}) \\
(5)\end{array}$ & $\begin{array}{c}\left\langle d_{\text {proj }}\right\rangle \\
(\mathrm{pc}) \\
(6)\end{array}$ & $\begin{array}{c}\langle\vartheta\rangle \\
(\mathrm{deg}) \\
(7)\end{array}$ & $\begin{array}{c}\phi \\
\text { (deg) } \\
(8)\end{array}$ & $\begin{array}{c}|\langle\vartheta\rangle-\phi| \\
(\operatorname{deg}) \\
(9)\end{array}$ & $\begin{array}{c}\mu \\
\left(\mu \operatorname{as~yr}^{-1}\right) \\
(10)\end{array}$ & $\begin{array}{c}\beta_{\text {app }} \\
(c) \\
(11)\end{array}$ & $\begin{array}{c}T_{\mathrm{ej}} \\
(12)\end{array}$ & $\begin{array}{l}T_{\text {mid }} \\
(13)\end{array}$ & $\begin{array}{c}\Delta \alpha \\
(\mu \text { as }) \\
(14)\end{array}$ & $\begin{array}{c}\Delta \delta \\
(\mu \text { as }) \\
(15)\end{array}$ \\
\hline \multirow[t]{5}{*}{$0003+380$} & 1 & 8 & 5 & 4.2 & 15.24 & 120.8 & $99 \pm 19$ & $22 \pm 19$ & $180 \pm 58$ & $2.62 \pm 0.84$ & & 2008.35 & 212 & 223 \\
\hline & 2 & 7 & 17 & 1.8 & 6.53 & 112.6 & $119.7 \pm 2.6$ & $7.1 \pm 2.7$ & $319 \pm 22$ & $4.63 \pm 0.32$ & $2003.01 \pm 0.24$ & 2007.70 & 64 & 24 \\
\hline & 3 & 5 & 17 & 1.3 & 4.72 & 114.2 & $202 \pm 15$ & $88 \pm 15^{\mathrm{a}}$ & $46 \pm 14$ & $0.67 \pm 0.20$ & & 2009.08 & 26 & 31 \\
\hline & 4 & 7 & 40 & 0.8 & 2.90 & 118.2 & $254 \pm 28$ & $135 \pm 28$ & $25 \pm 22$ & $0.36 \pm 0.32$ & & 2008.72 & 70 & 33 \\
\hline & $5^{\mathrm{b}}$ & 8 & 111 & 0.4 & 1.45 & 116.6 & $336 \pm 102$ & $140 \pm 102$ & $11 \pm 18$ & $0.16 \pm 0.26$ & & 2008.35 & 73 & 64 \\
\hline \multirow[t]{7}{*}{$0003-066$} & 2 & 5 & 222 & 1.0 & 4.88 & 322.9 & $226.3 \pm 4.8$ & $96.6 \pm 5.0^{\mathrm{a}}$ & $191 \pm 15$ & $4.09 \pm 0.33$ & & 1997.80 & 19 & 81 \\
\hline & 3 & 9 & 119 & 2.8 & 13.65 & 296.9 & $284.8 \pm 4.5$ & $12.1 \pm 4.6$ & $250 \pm 39$ & $5.35 \pm 0.83$ & & 1999.33 & 285 & 122 \\
\hline & 4 & 23 & 129 & 6.6 & 32.19 & 285.4 & $269.7 \pm 2.8$ & $15.6 \pm 2.8^{\mathrm{a}}$ & $79.9 \pm 6.2^{\mathrm{c}}$ & $1.71 \pm 0.13$ & $\ldots$ & 2003.87 & 113 & 70 \\
\hline & 5 & 6 & 1031 & 0.7 & 3.41 & 14.5 & $343.1 \pm 3.1$ & $31.4 \pm 3.1^{\mathrm{a}}$ & $100 \pm 16$ & $2.15 \pm 0.35$ & $\ldots$ & 2005.37 & 4 & 32 \\
\hline & 6 & 10 & 97 & 1.0 & 4.88 & 290.2 & $211.3 \pm 8.8$ & $78.9 \pm 8.9^{\mathrm{a}}$ & $54 \pm 11^{\mathrm{c}}$ & $1.16 \pm 0.24$ & $\ldots$ & 2003.78 & 32 & 69 \\
\hline & 8 & 9 & 105 & 2.2 & 10.73 & 294.6 & $292.9 \pm 2.6$ & $1.8 \pm 2.7$ & $392 \pm 18$ & $8.39 \pm 0.39$ & $2003.16 \pm 0.27$ & 2008.96 & 77 & 75 \\
\hline & 9 & 9 & 115 & 1.6 & 7.80 & 288.2 & $300.5 \pm 4.0$ & $12.2 \pm 4.1$ & $316 \pm 24$ & $6.78 \pm 0.52$ & $\ldots$ & 2008.96 & 105 & 86 \\
\hline $0007+106$ & 1 & 8 & 256 & 0.5 & 0.82 & 290.7 & $291.6 \pm 2.0$ & $0.9 \pm 2.4$ & $204 \pm 12$ & $1.196 \pm 0.069$ & $2003.99 \pm 0.16$ & 2006.77 & 60 & 28 \\
\hline \multirow[t]{2}{*}{$0010+405$} & 1 & 10 & 3 & 8.2 & 32.35 & 328.7 & $340.8 \pm 4.1$ & $12.1 \pm 4.1$ & $428 \pm 40$ & $6.91 \pm 0.64$ & $\cdots$ & 2008.52 & 141 & 198 \\
\hline & $2^{\mathrm{b}}$ & 11 & 10 & 1.7 & 6.71 & 328.6 & $354 \pm 111$ & $26 \pm 111$ & $6 \pm 19$ & $0.10 \pm 0.30$ & $\ldots$ & 2008.62 & 62 & 99 \\
\hline
\end{tabular}

Notes. The kinematic fit values are derived from the acceleration fit for components with significant acceleration, and from the vector motion fit otherwise. Columns are as follows: (1) B1950 name, (2) component number, (3) number of fitted epochs, (4) mean flux density at $15 \mathrm{GHz}$ in mJy, (5) mean distance from core component in mas, (6) mean projected distance from core component in pc, (7) mean position angle with respect to the core component in degrees, (8) position angle of velocity vector in degrees, (9) offset between mean position angle and velocity vector position angle in degrees, (10) angular proper motion in microarcseconds per year, (11) fitted speed in units of the speed of light, (12) fitted ejection date, (13) date of reference (middle) epoch used for fit, (14) right ascension error of individual epoch positions in $\mu$ as, and (15) declination error of individual epoch positions in $\mu$ as. A question mark indicates a non-radially moving component for which an inward/outward determination is uncertain due to its slow angular speed.

${ }^{a}$ Component shows significant non-radial motion.

${ }^{\mathrm{b}}$ Component has a slow pattern speed.

${ }^{\mathrm{c}}$ Component shows significant accelerated motion.

${ }^{\mathrm{d}}$ Component shows significant inward motion.

(This table is available in its entirety in machine-readable and Virtual Observatory (VO) forms in the online journal. A portion is shown here for guidance regarding its form and content.) 
Table 6

Acceleration Fit Properties of Jet Components

\begin{tabular}{|c|c|c|c|c|c|c|c|}
\hline $\begin{array}{l}\text { Source } \\
\text { (1) }\end{array}$ & $\begin{array}{l}\text { ID } \\
(2)\end{array}$ & $\begin{array}{c}\phi \\
(\mathrm{deg}) \\
(3)\end{array}$ & $\begin{array}{c}\mu \\
\left(\mu{\operatorname{as~} \mathrm{yr}^{-1}}^{-1}\right) \\
(4)\end{array}$ & $\begin{array}{c}\dot{\mu}_{\perp} \\
\left(\mu \text { as } \mathrm{yr}^{-2}\right) \\
(5)\end{array}$ & $\begin{array}{c}\dot{\mu}_{\|} \\
\left(\mu \text { as } \mathrm{yr}^{-2}\right) \\
(6)\end{array}$ & $\begin{array}{l}\dot{\eta}_{\perp} \\
\text { (7) }\end{array}$ & $\begin{array}{l}\dot{\eta}_{\|} \\
(8)\end{array}$ \\
\hline \multirow[t]{2}{*}{$0003-066$} & $4^{\mathrm{a}}$ & $269.7 \pm 2.8$ & $79.9 \pm 6.2$ & $9.8 \pm 1.9$ & $-24.1 \pm 3.1$ & $0.165 \pm 0.035$ & $-0.407 \pm 0.061$ \\
\hline & $6^{\mathrm{a}}$ & $211.3 \pm 8.8$ & $54 \pm 11$ & $54 \pm 11$ & $-37 \pm 15$ & $\ldots$ & $\ldots$ \\
\hline \multirow[t]{4}{*}{$0010+405$} & 1 & $340.7 \pm 4.3$ & $428 \pm 40$ & $11 \pm 53$ & $-43 \pm 69$ & $0.03 \pm 0.16$ & $-0.13 \pm 0.20$ \\
\hline & 2 & $354 \pm 132$ & $6 \pm 19$ & $1 \pm 20$ & $4 \pm 33$ & $\ldots$ & $\ldots$ \\
\hline & 3 & $181 \pm 258$ & $1.6 \pm 4.4$ & $-8.2 \pm 6.5$ & $-2 \pm 10$ & $\ldots$ & $\ldots$ \\
\hline & 4 & $120 \pm 103$ & $2.4 \pm 3.5$ & $-2.5 \pm 8.8$ & $-3.5 \pm 7.1$ & $\ldots$ & $\ldots$ \\
\hline $0016+731$ & $1^{\mathrm{a}}$ & $163.2 \pm 2.2$ & $106.2 \pm 4.4$ & $10.2 \pm 1.8$ & $8.8 \pm 1.9$ & $0.266 \pm 0.048$ & $0.231 \pm 0.051$ \\
\hline \multirow[t]{7}{*}{$0055+300$} & 4 & $304 \pm 17$ & $37 \pm 11$ & $6.8 \pm 7.3$ & $-1.8 \pm 8.0$ & $\ldots$ & $\ldots$ \\
\hline & $6^{\mathrm{a}}$ & $270 \pm 11$ & $24.5 \pm 3.0$ & $7.6 \pm 3.0$ & $6.7 \pm 2.0$ & $\ldots$ & $\ldots$ \\
\hline & 10 & $321.6 \pm 5.5$ & $46.9 \pm 4.1$ & $-0.8 \pm 2.7$ & $0.9 \pm 2.6$ & $\ldots$ & $\ldots$ \\
\hline & 12 & $-0 \pm 44$ & $2.9 \pm 2.5$ & $2.7 \pm 1.3$ & $-3.7 \pm 1.3$ & $\ldots$ & $\ldots$ \\
\hline & 13 & $-0 \pm 39$ & $3.9 \pm 2.6$ & $1.7 \pm 1.7$ & $-3.4 \pm 1.2$ & $\ldots$ & $\ldots$ \\
\hline & 14 & $-0 \pm 32$ & $2.9 \pm 1.9$ & $1.96 \pm 0.86$ & $-2.9 \pm 1.0$ & $\ldots$ & $\ldots$ \\
\hline & 15 & $336 \pm 66$ & $1.1 \pm 1.4$ & $-0.47 \pm 0.79$ & $-0.55 \pm 0.86$ & $\ldots$ & $\ldots$ \\
\hline $0059+581$ & 2 & $257.4 \pm 3.0$ & $168 \pm 14$ & $8.3 \pm 5.5$ & $-16.5 \pm 8.2$ & $0.081 \pm 0.054$ & $-0.161 \pm 0.081$ \\
\hline
\end{tabular}

Notes. Columns are as follows: (1) B1950 name, (2) component number, (3) proper-motion position angle in degrees, (4) angular proper motion in microarcseconds per year, (5) angular acceleration perpendicular to velocity direction in microarcseconds per year per year, (6) angular acceleration parallel to velocity direction in microarcseconds per year per year, (7) relative acceleration in direction perpendicular to fitted velocity vector, and (8) relative acceleration in direction parallel to fitted velocity vector.

${ }^{a}$ Component shows significant accelerated motion.

(This table is available in its entirety in machine-readable and Virtual Observatory (VO) forms in the online journal. A portion is shown here for guidance regarding its form and content.)

Paper VI), which yielded kinematic quantities at a reference (middle) epoch. The results of this analysis are listed in Tables 5 and 6 . Table 5 gives the mean flux density, core separation distance and position angle, and the best-fit proper-motion vector and reference epoch for each robust component. For components that show significant $(\geqslant 3 \sigma)$ accelerations, the values listed in Table 5 are from the acceleration fit.

Figure 3 shows the radial separation of the components from the core component over time for each jet. The left panels of Figure 4 show the motions of individual components on the sky, as well as a $15 \mathrm{GHz}$ VLBA contour image of the jet at the middle epoch listed in Table 5. The orange box delimits the zoomed region displayed in the right panels. Extended versions of these figures, containing plots of all of the robust jet components, are available online.

We examined each component with significant proper motion $\left(\mu \geqslant 3 \sigma_{\mu}\right)$ for non-radial motion by comparing the mean position angle of the component $\langle\vartheta\rangle$ with its proper-motion vector direction $\phi$. We flagged any component for which the angular offset $|\langle\vartheta\rangle-\phi|$ was $\geqslant 3 \sigma$ from either $0^{\circ}$ or $180^{\circ}$ as "non-radial," and "inward" if the offset was significantly greater than $90^{\circ}$. We made these determinations using the $|\langle\vartheta\rangle-\phi|$ values from the acceleration fit for significantly accelerating components, or from the vector fits otherwise. Of the $739(\geqslant 3 \sigma)$ motion components classified as robust, $282(38 \%)$ exhibit significant non-radial motion, while only $17(2.3 \%)$ are flagged as inward (see Table 7). We discuss the rare inward motion cases in Section 5.1.

We calculated the ejection times (defined as when the calculated core separation equals zero) for the non-accelerating, non-inward components by taking the average value extrapolated from the proper-motion fits in the right ascension and declination directions. We did not compute ejection times for components that had significant vector motion offsets (within $2 \sigma$ of $15^{\circ}$ or larger), since this would involve an extrapolation of
Table 7

Summary of Kinematics Analysis Results

\begin{tabular}{lc}
\hline \hline Property & Total Number \\
\hline AGNs analyzed for kinematics & 200 \\
Jet components classified as robust & 887 \\
Slow pattern speed components & $38(4 \%)$ \\
Jet components with $(\geqslant 3 \sigma)$ measured speeds & $739(83 \%)$ \\
Inward-moving $(\geqslant 3 \sigma$ speed) components & $17(2 \%)$ \\
Components with significant $(\geqslant 3 \sigma)$ non-radial motion & $282(38 \%)$ \\
Components analyzed for acceleration & $547(62 \%)$ \\
Components with significant acceleration & $212(39 \%)$ \\
Components with significant perpendicular acceleration & $99(18 \%)$ \\
Components with significant parallel acceleration & $155(28 \%)$ \\
\hline
\end{tabular}

an unknown acceleration. The errors on the ejection times were calculated by the same method as Paper VI; we note that the numerical $t_{\mathrm{ej}}$ error values tabulated in that paper were a factor of $\sqrt{2}$ too large due to a calculation error.

We list the parameters of the acceleration fits in Table 6, where we have resolved the acceleration terms $\dot{\mu}_{\perp}$ and $\dot{\mu}_{\|}$ in directions perpendicular and parallel, respectively, to the mean angular velocity direction $\phi$. For those components with a measured redshift, a significant proper motion $\left(\mu \geqslant 3 \sigma_{\mu}\right)$, and a small uncertainty in the radial motion offset $\left(|\langle\vartheta\rangle-\phi| \leqslant 5^{\circ}\right)$, we calculate relative accelerations as in Paper VII, where $\dot{\eta}=(1+z) \dot{\mu} / \mu$.

\section{DISCUSSION}

\subsection{Apparent Inward Motions}

There are many scenarios under which apparent inward motions can be produced, including: (1) curved jet motions that cross the line of sight; (2) non-stationarity of the apparent core feature, due to one or more newly emerging features below 


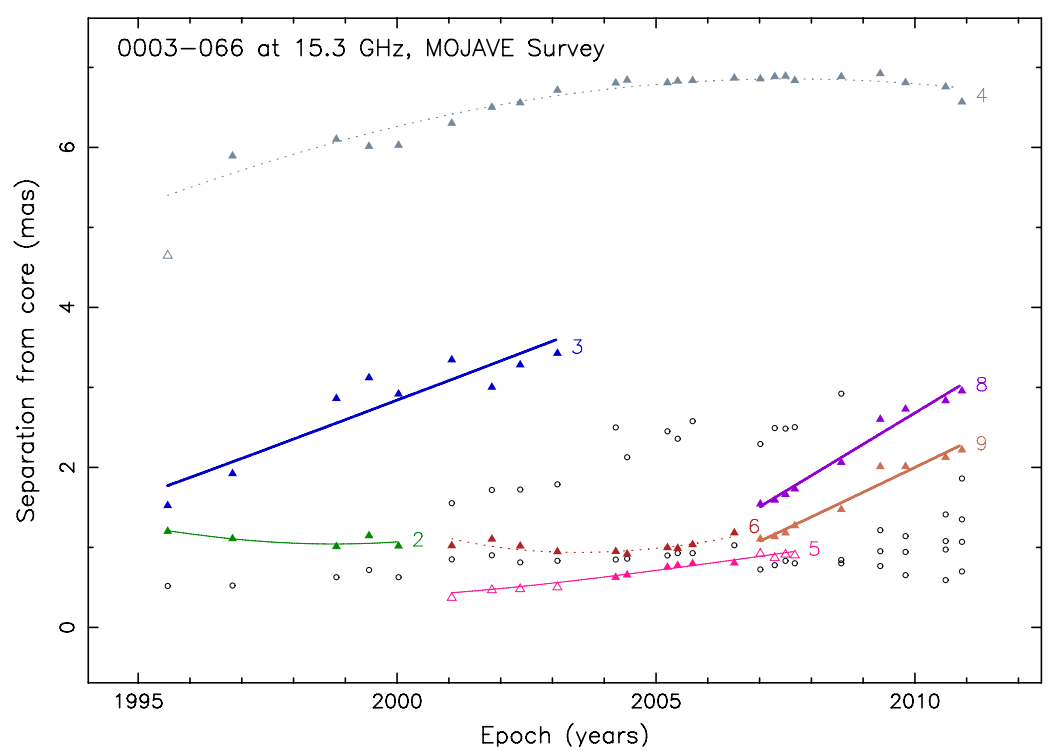

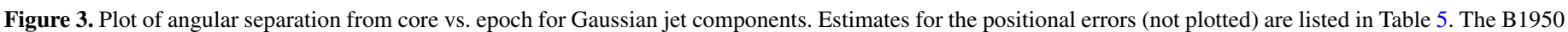

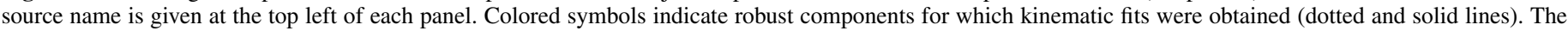

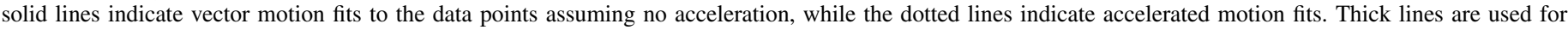

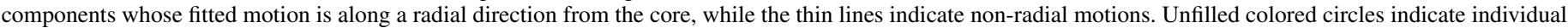

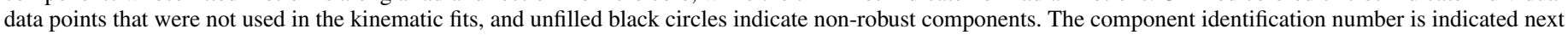
to the last epoch of each robust component.

(A color version and the complete figure set (200 images) are available in the online journal.)

the interferometric resolution level; (3) a misidentification of the true stationary core with a moving feature; (4) internal brightness changes in a large, diffuse jet feature; and (5) apparent backward pattern speeds not associated with the flow. In Paper VI we identified fewer than 10 individual cases of inward motion, which represented $<2 \%$ of the robust components. This strongly ruled out a fully random pattern speed scenario for the component motions, in which equal numbers of inward and outward motions would be expected.

Among all 887 robust components which we have analyzed, we find 17 statistically significant inward component motions in 10 different jets (6 BL Lac objects, 2 quasars, and 2 radio galaxies). BL Lac jets are statistically overrepresented in this group, given that they make up only $20 \%$ of the 200 AGN jets we analyzed for kinematics. We do not find anything otherwise distinctive about these particular BL Lac objects, and the small number statistics make it impossible to draw any firm conclusions as to the cause of this overrepresentation. We also note that 4 of the 10 inward component motion jets are BL Lac objects with no measured redshift. The latter is not unexpected, however, since by definition BL Lac objects have weak emission lines or featureless optical spectra that often make it difficult or impossible to measure their redshifts.

We previously identified three jets $(1458+718,2021+614$, and 2230+114) as having apparent inward motions in Paper VI. With the addition of our new data, the jets of 2005+403, 2200+420 (BL Lac), 2201+171, and 2351+456 no longer display any statistically significant inward motions (see Appendix for details).

The inward motions are all typically slow, with a median

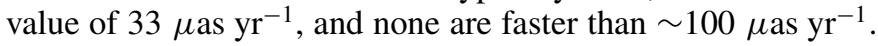
Considering only the AGN with a known redshift, the inward components of $1458+718$ are the only ones which appear significantly superluminal, ranging from $1.4 \mathrm{c}$ to $4.6 \mathrm{c}$. With the exception of $1458+718,2021+614$, and $2230+114$, the inward motions all occur within $\sim 1$ mas of the core, in typically the innermost component. In particular, the innermost two jet components of two TeV-emitting BL Lac objects in our sample, 0219+428 (3C 66A) and 1219+285 (W Comae), are both inward moving. The small velocities and core separations of these moving components may indicate that the core is not a stable reference point in these two jets. We did not find any significant inward motions in the other 15 currently known TeV-emitting AGN jets ${ }^{12}$ in Table 1 which we analyzed.

\subsection{Parsec-scale Jet Orientation Variations}

The tendency for the parsec-scale jets of blazars to change their position angles on the sky with time has been solidly established via long-term VLBI studies of several individual AGNs (see Agudo 2009 for a recent review). The exact origin of the wobbling is not clear, although accretion disk precession, orbital motion of the accretion system, or instabilities in the jet flow have all been suggested. A main signature of precession is sinusoidal variations in the jet position angle, and evidence for this has been seen in blazars such as 3C 273 (Savolainen et al. 2006), 3C 345 (Lobanov \& Roland 2005), 0716+714 (Bach et al. 2005), BL Lac (Stirling et al. 2003), and M81 (Marti-Vidal et al. 2013). Other jets have displayed monotonic position angle swings with no evidence of periodicity (e.g., 3C 279, Jorstad et al. 2004; NRAO 150, Agudo et al. 2007).

Until now, there has been no systematic survey of jet position angle variations in a large blazar sample. Using our extensive MOJAVE database, we have analyzed the innermost jet regions of 60 AGNs from our radio-selected sample for which we have obtained 20 or more VLBA epochs over a minimum $12 \mathrm{yr}$ period. We excluded several well-monitored jets in our sample for reasons of core identification uncertainty, counterjet emission, or highly curved jet structure within 1 mas of the core. We determined the innermost jet position angle at each epoch by taking a flux-density-weighted position angle average of all clean components above three times the image noise level in the annular region from 0.15 mas to 1 mas from the

\footnotetext{
12 http://tevcat.uchicago.edu
} 

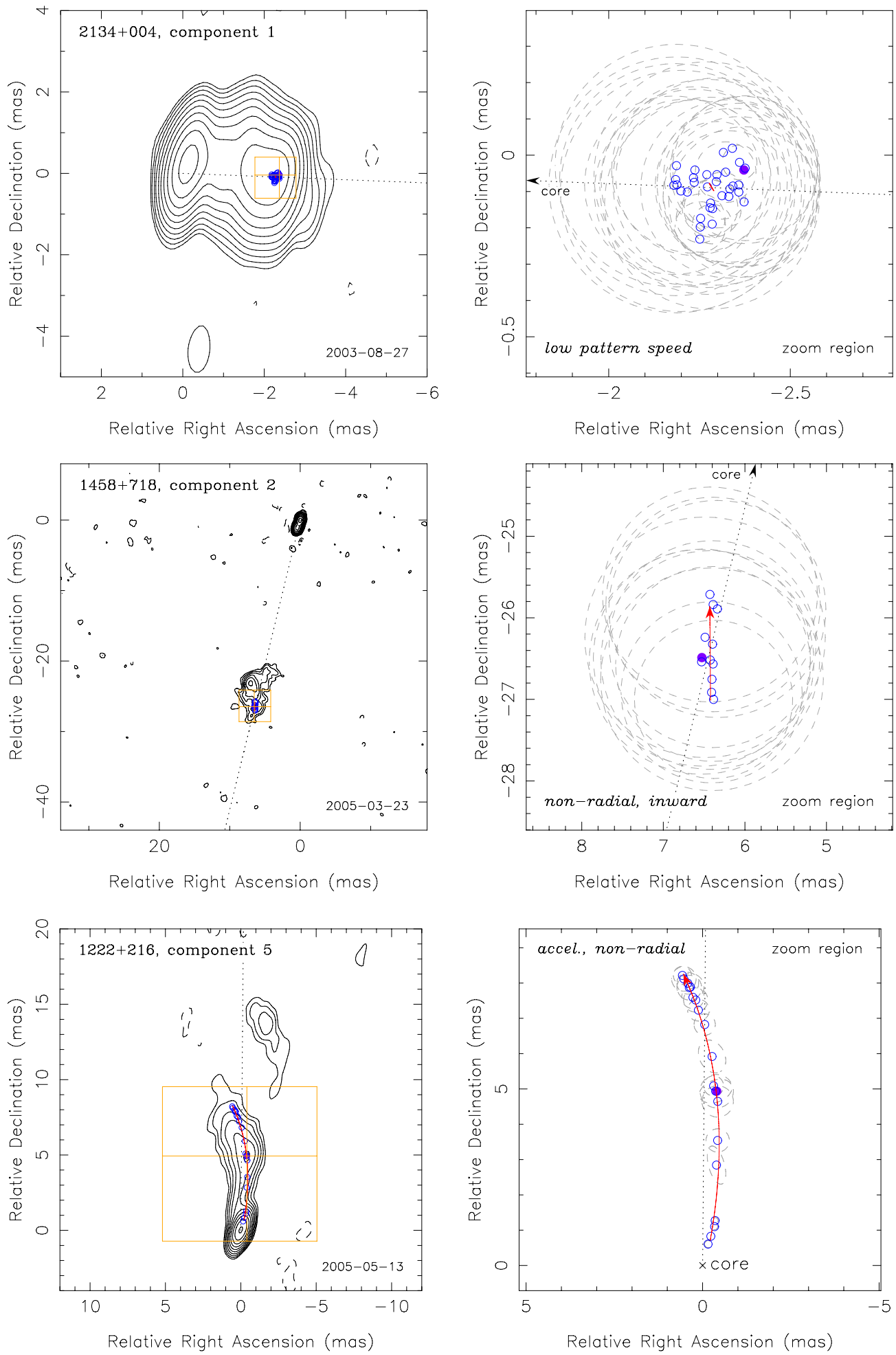

Figure 4. Vector motion fits and sky position plots of individual robust jet components in MOJAVE AGNs. Positions are relative to the core position. The left panels show a $15 \mathrm{GHz}$ VLBA contour image of the jet at the middle epoch listed in Table 5. The orange box delimits the zoomed region displayed in the right panels. The component's position at the middle epoch is indicated by the orange cross-hairs. The dotted line connects the component with the core component and is plotted with the mean position angle $\langle\vartheta\rangle$ (Table 5). The position at the middle epoch is shown by a filled violet circle, while other epochs are plotted with unfilled blue circles. The red solid line indicates the vector (or accelerating) fit (see Table 5) to the component positions. The red arrows in the right panels indicate the direction of motion, and the gray dashed circles/ellipses represent the FWHM sizes of the individual fitted Gaussian components. Displayed from top to bottom in the figure are component $\mathrm{ID}=1$ in $2134+004, \mathrm{ID}=2$ in $1458+718, \mathrm{ID}=5$ in $1222+216, \mathrm{ID}=2$ in $2251+158, \mathrm{ID}=2$ in $1828+487$, and ID = 16 in $0716+714$.

(A color version and the complete figure set (887 images) are available in the online journal.) 

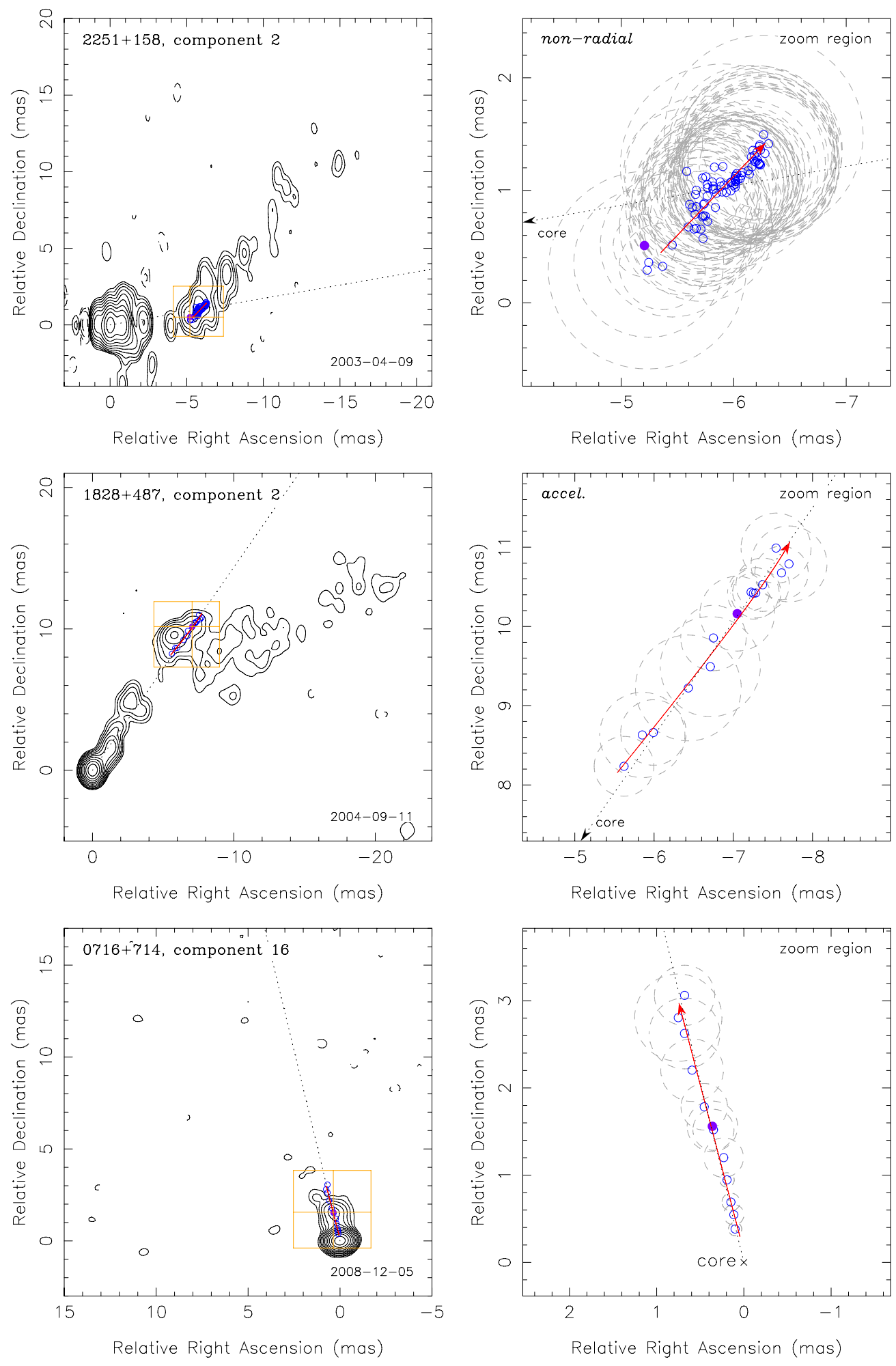

Figure 4. (Continued)

core. We also explored other methods, such as using the position angle of the innermost Gaussian model fit component, or multiple components within a particular distance from the core, but the derived position angles were more influenced by choices made for the fitted Gaussians (e.g., elliptical versus circular, and total number of Gaussians in the inner jet region). Our position angle measurement method is subject to errors associated with wandering of the core component position due to changes in opacity or the emergence of new components below our resolution level. These are likely small, however, since they would create correlated apparent motions in components located downstream, which we have not detected in our data.

We find that the innermost jet position angles vary considerably over the 12-16 yr intervals covered by our data, with ranges up to $150^{\circ}$ in some jets (Figure 5). The typical circular 

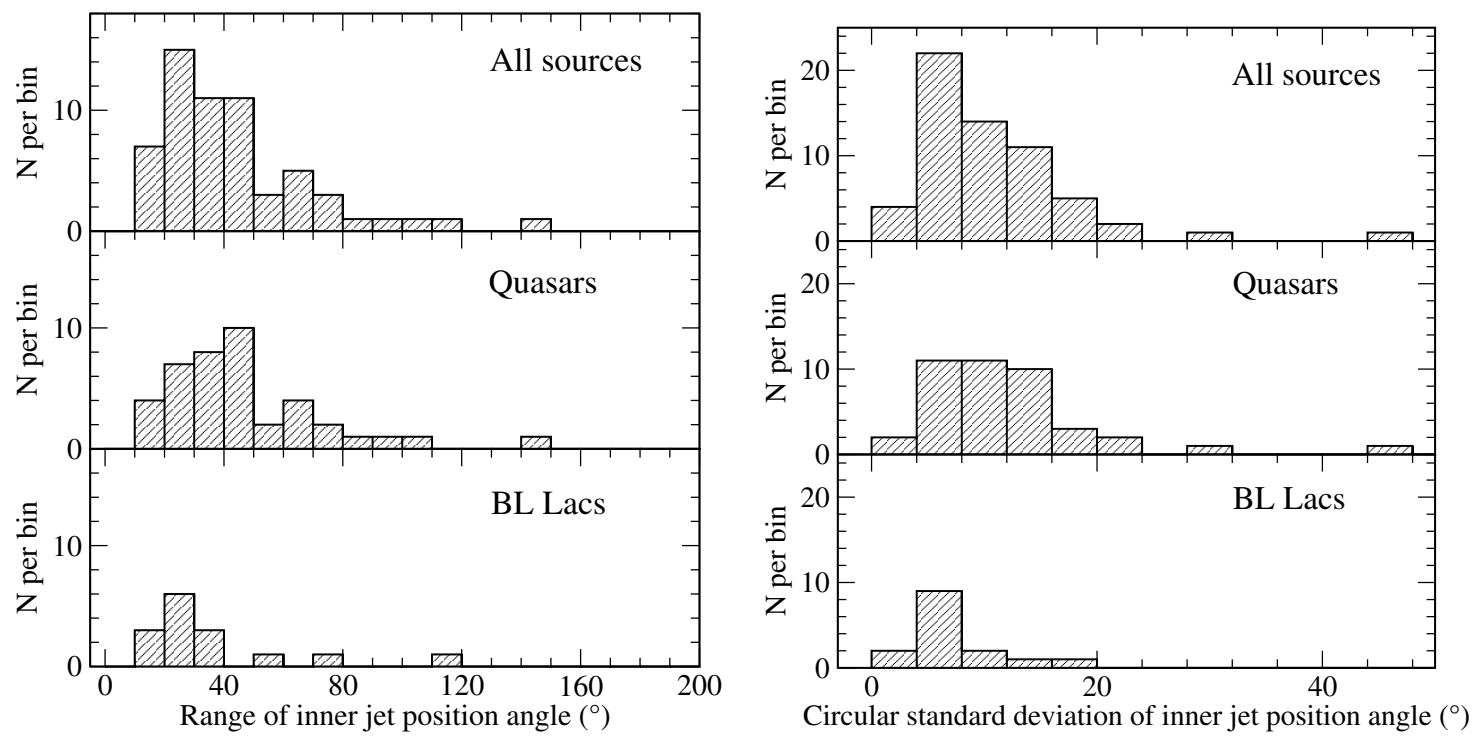

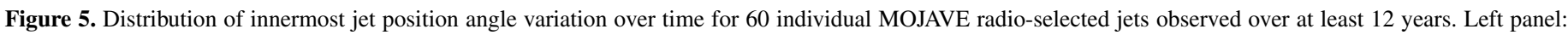

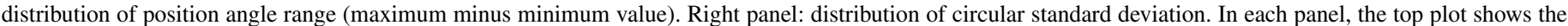

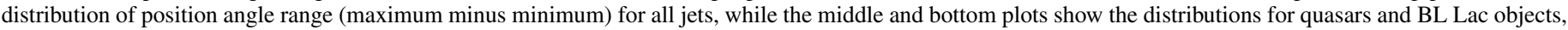
respectively.

standard deviation in position angle is $\sim 10^{\circ}$. The quasars and BL Lac objects differ significantly in their ranges, according to Kolmogorov-Smirnov tests on their range $\left(p_{\text {null }}=1.1 \%\right)$ and standard deviation ( $p_{\text {null }}=1.3 \%$ ) distributions. It is unclear whether the smaller overall variations we see in the BL Lac innermost jet position angles are an intrinsic effect or a result of them being oriented at slightly larger angles to the line of sight than quasars.

Some jets (e.g., NRAO $150=0355+508$ ) show a very wide range of inner jet position angle and Gaussian component position angles, likely because the viewing angle to the inner jet lies within the opening angle of the (presumably conical) outflow. In other cases, such as 3C $273(1226+023)$, the jet is transversely resolved into multiple features, some of which are moving along different position angles at nearly the same radial distance from the core. Finally, the distributions shown in Figure 5 are not peaked in the first bin, implying that it is common in blazars for features to emerge at different position angles. Since stacked-epoch VLBA images (Paper V) often show a smooth conical jet intensity profile in highly variable ejection angle jets such as $1308+326$, the simplest interpretation is that individual emerging features do not fill the entire cross section of the flow. Instead, features are ejected within a finite width "ejection cone," which becomes apparent only in a stacked-epoch image. The apparent opening angle of this cone is exaggerated by projection effects by a factor of $\sin \theta$. Thus, for typical blazar jet viewing angles of $\theta \lesssim 5^{\circ}$, the intrinsic ejection cone full opening angle is likely $\sim 0^{\circ} .5$ to $\sim 2^{\circ}$, based on the position angle range distribution in Figure 5 . AGN jets can therefore appear "bent" in a single-epoch, limited dynamic range VLBI image, whereas in reality what is visible is just the portion of the jet that is currently experiencing enhanced synchrotron emission, due to the passage of several very bright features. These features may represent flow instabilities driven at the nozzle (e.g., Hardee 2011), which vary within the jet over time and in turn influence the kinematics downstream.

In Figure 6 we plot the innermost jet position angle derived from the clean components for each jet versus time on the same vertical scale. The individual jets show a variety of behavioral
Table 8

Linear Regression Fits to Monotonic Innermost Jet Position Angle Trends

\begin{tabular}{|c|c|c|}
\hline $\begin{array}{l}\text { Source } \\
\text { Name }\end{array}$ & $\begin{array}{c}\text { Slope } \\
\left(\operatorname{deg} \mathrm{yr}^{-1}\right)\end{array}$ & $\begin{array}{c}\text { Fit } \\
\text { Range }\end{array}$ \\
\hline $0355+508$ & $9.8 \pm 1$ & 1995.1-2010.8 (all data) \\
\hline $0607-157$ & $4.6 \pm 0.9$ & 1995.6-2009.5 (all data) \\
\hline $0748+126$ & $2.2 \pm 0.4$ & 1997.0-2011.0 data only \\
\hline $0851+202$ & $-2.6 \pm 0.1$ & 1995.3-2010.0 data only \\
\hline $0906+015$ & $-0.7 \pm 0.2$ & 1997.6-2011.2 (all data) \\
\hline $1226+023$ & $-1.9 \pm 0.2$ & 1995.6-2010.8 (all data) \\
\hline $1253-055$ & $-1.5 \pm 0.1$ & 1995.6-2007.0 data only \\
\hline $1633+382$ & $2.2 \pm 0.2$ & 1995.1-2011.1 (all data) \\
\hline $1807+698$ & $0.4 \pm 0.1$ & 1996.0-2011.1 data only \\
\hline $2005+403$ & $3.8 \pm 0.7$ & 1995.1-2011.1 (all data) \\
\hline $2200+520$ & $-0.8 \pm 0.2$ & 1995.3-2011.3 (all data) \\
\hline $2223-052$ & $2.8 \pm 0.5$ & 1995.6-2010.7 (all data) \\
\hline $2230+114$ & $2.1 \pm 0.4$ & 1998.5-2010.8 data only \\
\hline $2251+158$ & $-2.0 \pm 0.1$ & 1995.6-2011.3 (all data) \\
\hline
\end{tabular}

patterns, which we have classified into four general categories. There are 14 jets that display a monotonic trend, 5 that show a back and forth trend, 12 that show more than one cycle of back and forth motion (oscillatory), and 29 with no discernible trend. Within these categories, 11 jets exhibited one or more abrupt jumps in position angle, caused by a new feature emerging from the core with a significantly different trajectory than previously ejected features.

We performed linear regression fits on the jets showing monotonic trends and tabulated the fitted slopes in Table 8 . In some cases, we had to omit segments of the data from the fit due to abrupt jumps in position angle (see Column 3 of Table 8). The most rapidly varying jet, at $9.8 \pm 1^{\circ} \mathrm{yr}^{-1}$, is $0355+508$ (NRAO 150). Our measurement is consistent with the swings of up to $11^{\circ} \mathrm{yr}^{-1}$ seen previously in this jet by Agudo et al. (2007) in VLBA images at 43 and $86 \mathrm{GHz}$. The other monotonically changing jets in our sample show swings of typically a few degrees per year. In the particular case of $0851+202$ (OJ 287), Agudo et al. (2012) found erratic variations in the innermost jet position angle at $43 \mathrm{GHz}$, with amplitudes $<40^{\circ}$ 

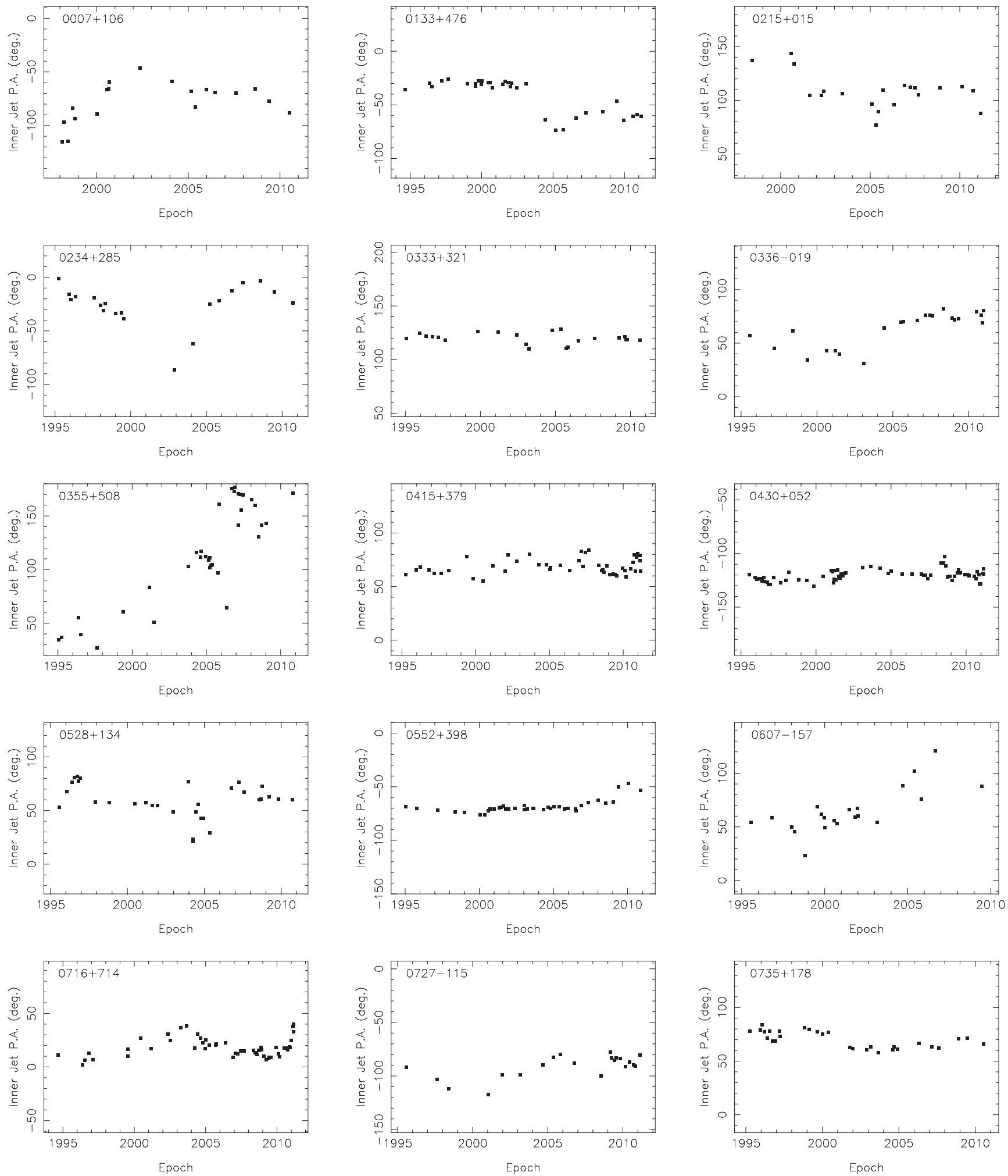

Figure 6. Innermost jet position angle vs. time plots for 60 selected MOJAVE jets with 20 or more VLBA epochs over at least 12 years.

(The complete figure set (60 images) is available in the online journal.)

and timescales $\lesssim 2 \mathrm{yr}$, as well as an abrupt jump in position angle in late 2004. Our observations at $15 \mathrm{GHz}$, on the other hand, show a monotonic swing in position angle of $-2.6 \pm 0.1 \mathrm{yr}^{-1}$ from 1995 until the end of 2010, when the jet underwent a sudden large jump in position angle. Earlier VLBI observations, as tabulated by Valtonen \& Wiik (2012), show that this monotonic swing began in the early 1990s. Superimposed on the long-term trend are small-amplitude wiggles with timescales of several years (Figure 6). The 2004 jump seen at $43 \mathrm{GHz}$ is not present in the $15 \mathrm{GHz}$ data, and the 2010 jump seen at $15 \mathrm{GHz}$ is not evident in the $43 \mathrm{GHz}$ data. This likely reflects the different angular resolution of the two data sets. Agudo et al. (2012) 

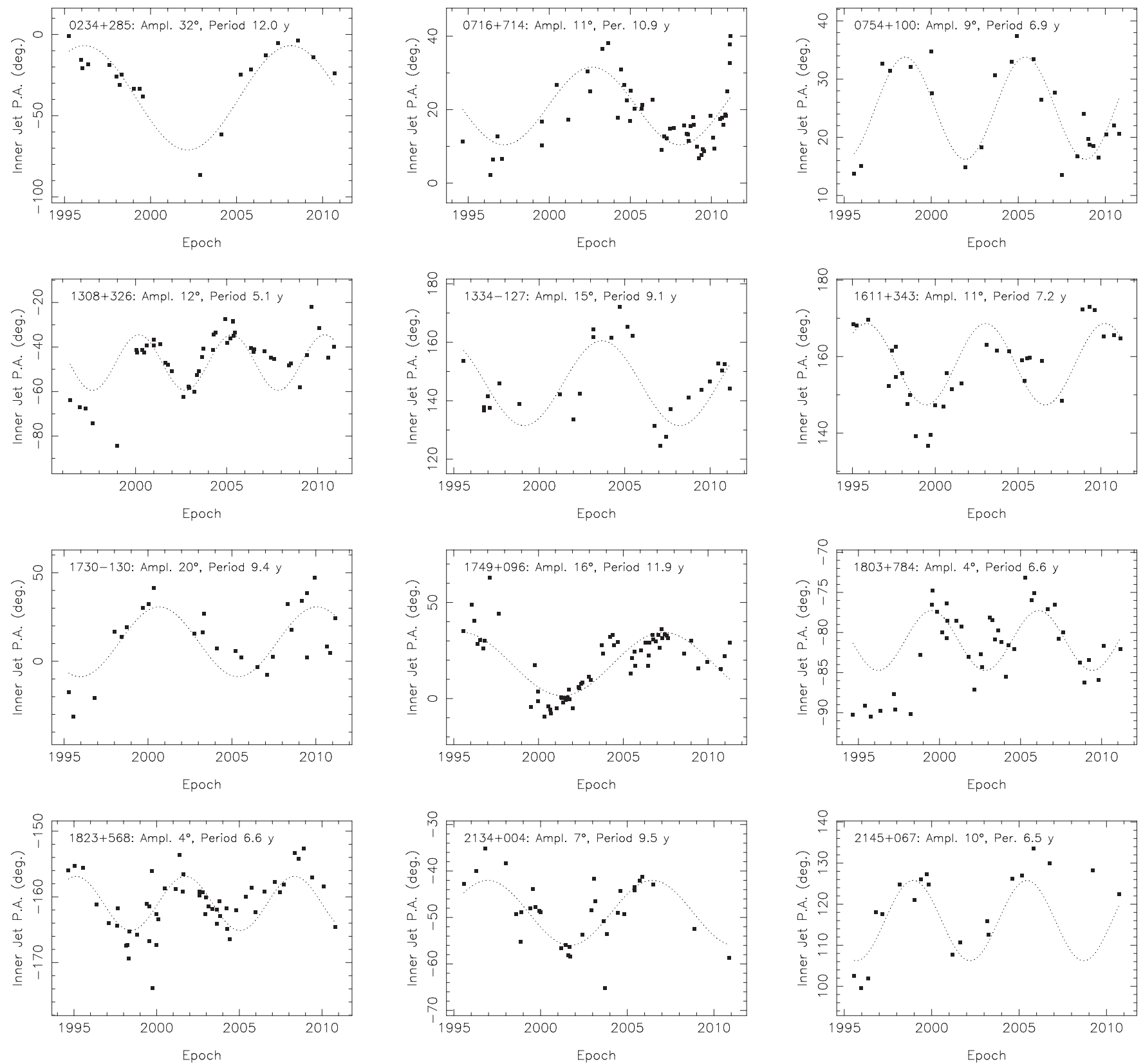

Figure 7. Innermost jet position angle vs. time plots for 12 selected MOJAVE jets displaying oscillatory trends. The dotted lines represent the best sine curve fits to the data, based on the peak period in the Lomb-Scargle periodogram.

used the position angle of Gaussian components out to $\sim 0.4$ mas from the core, with no flux density weighting, whereas at $15 \mathrm{GHz}$ we used a flux-density-weighted average of clean components in the inner $0.15-1$ mas region. It is also possible that OJ 287 emits very short-lived, bright features visible at $43 \mathrm{GHz}$ that rapidly fade before they can be resolved in our $15 \mathrm{GHz}$ images.

We looked for evidence of periodicity in all 60 jets using Lomb-Scargle periodograms, which are well suited for unequally sampled time-series data (Lomb 1976; Scargle 1982). With the exception of $0234+285$ and $2145+067$, the 12 jets shown in Figure 7 have significant $(\geqslant 2 \sigma)$ peaks at the periods indicated on each sub-panel, which range from 5 to $12 \mathrm{yr}$. The dashed lines represent the best sinusoid fits to the data for the indicated Lomb-Scargle period. For these fits we allowed the amplitude, mean, and phase to vary in order to find the best absolute $\chi^{2}$ value. Assuming a typical Gaussian normal error of $2^{\circ}$ for the position angle measurements (based on the linear regression fit residuals to the monotonic trend sources), the best reduced- $\chi^{2}$ fit values are 1.5 for $0716+714$ and 2.5 for $1823+568$. We cannot reliably establish periodicity in any jet, however, due to the lack of sufficient cycles in the data and the fact that many of the fits have significant residuals (e.g., 1308+326, 1803+784, and 2145+067), suggesting that the behavior is more complex than a single sinusoidal variation.

\subsection{Dispersion of Apparent Speeds}

A long-standing question in the study of AGN jet kinematics is whether the bright features in a given jet all tend to propagate at a characteristic speed that represents the true flow. In Paper VI we found that roughly $20 \%$ of the jets had one or more features 

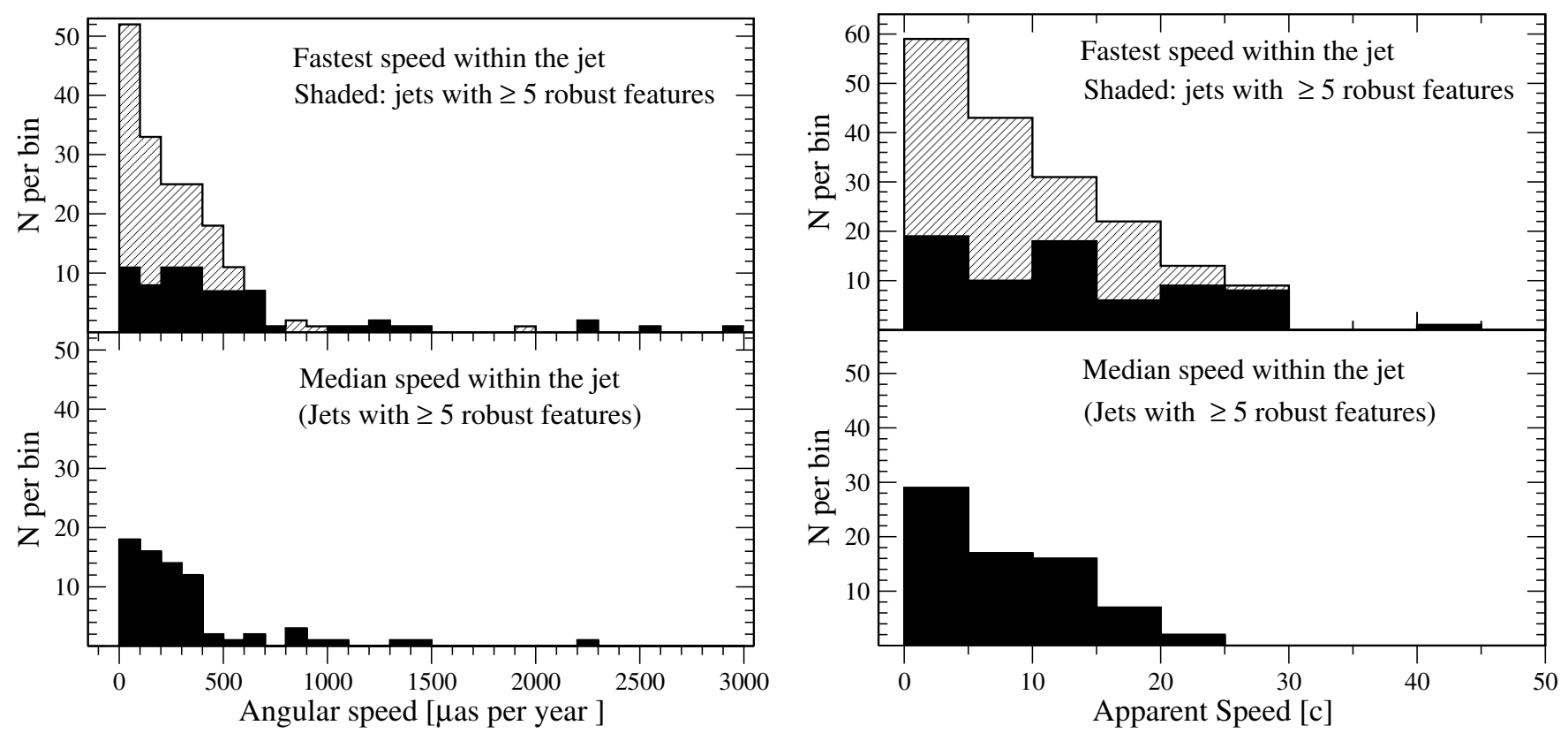

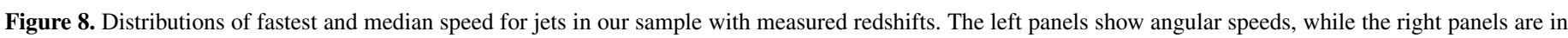
units of the speed of light. The shaded histograms are for jets with at least five robust components.

that moved significantly slower than the other features in the jet. These slow pattern speed (SPS) features could be the result of either stationary shocks in the flow or jet bending across the line of sight. We have repeated this analysis for the current data set, using more stringent criteria.

We first tabulated a maximum speed for each jet by considering the component with the fastest $\geqslant 3 \sigma$ speed. If no component in the jet had $\mathrm{a} \geqslant 3 \sigma$ speed, we lowered the criterion to $>2 \sigma$. In the case of $0355+508,1329-049$, and $1520+319$, which had no $\geqslant 2 \sigma$ components, we used the component speed with the smallest measured error. We dropped 16 jets from the analysis since they had no robust components with which to measure a maximum speed. We flagged components in Table 4 as SPS if they had (1) no statistically significant acceleration, (2) a speed less than $20 \mu \mathrm{as} \mathrm{yr}^{-1}$, and (3) a speed at least 10 times slower than the fastest component in the jet. We found 38 such components in 29 different jets (14 quasars, 10 BL Lac objects, and 4 radio galaxies).

A significantly higher fraction of BL Lac objects and radio galaxies in our sample contain SPS components $(\sim 25 \%)$ as compared to quasar jets (10\%). We detect no significant differences in the redshift distributions of SPS and non-SPS jets, but their median VLBA $15 \mathrm{GHz}$ luminosity distributions are different at the $98 \%$ confidence level according to a Kolmogorov-Smirnov test. Of the 37 jets with radio luminosity above $10^{28} \mathrm{~W} \mathrm{~Hz}^{-1}$, only one has an SPS component (the quasar 2134+004). This is consistent with numerical simulations (e.g., Duncan \& Hughes 1994; Rosen et al. 1999) which show that more highly relativistic jets exhibit fewer compact internal structures and less overall instability.

In order to examine the dispersion of speeds within individual jets in more detail, we calculated a normalized statistic $D=$ $\left(\mu_{\max }-\mu_{\min }\right) /\left(\mu_{\max }+\mu_{\min }\right)$ for all 75 jets that had at least five robust components, as well as a median speed and rms dispersion value. Over half of these jets have $D$ values above 0.8 , indicating that in most cases the overall range of apparent speed within a jet is comparable to its maximum speed. Removing the
SPS components from the analysis did not change this general result.

Although we find that there can be a large range of apparent speed within a jet, the speeds usually cluster around a median value, indicating they are not random. The median rms dispersion of speed within a jet is $2.8 c$. This is significantly less than the overall dispersion of the median values $(6.7 c)$. Furthermore, the distributions of maximum and median speed in the sample are not uniformly distributed in either angular or spatial measure, but are instead peaked at low values (Figure 8).

In Figure 9 we plot a representation of the apparent speed distribution within each of the 13 jets in the sample that have at least 10 robust components. For each component, we plot $\left(\mu-\mu_{\text {median }}\right) / \mu_{\text {median }}$, the fractional difference with respect to the median speed in the jet. The components in each jet are arranged vertically from slowest speed at the top to fastest speed at the bottom. We use this format instead of a binned representation in order to display the error bars. The binned distribution for the combined set of 13 jets is shown in Figure 10.

There is a range of behavior seen among these jets, with some (e.g., 0238-084, 0430+052, 1641+399, and 2200+420) having a near-Gaussian speed distribution, and others such as $1633+382$ and $1823+568$ ejecting features uniformly distributed over a wide range of speeds. Nevertheless, the combined distribution in Figure 10 shows a near-Gaussian shape, although with significant kurtosis (more sharply peaked than Gaussian, according to an Anscombe-Glynn test). The distribution has no significant skewness if the outlier at 2.8 is omitted (D'Agostino test). One might expect an asymmetric distribution (positively skewed) if some fraction of the features travel at the true flow speed and none of them exceed it, but we do not see evidence of this in the data. Since we can typically identify more robust components in the jets for which we have the longest monitoring intervals, we might also expect to see a positive trend between the maximum speed of a jet and the number of robust components, but this is not evident in our data. Our findings thus suggest that in general, high-ejection-rate blazar jets tend 


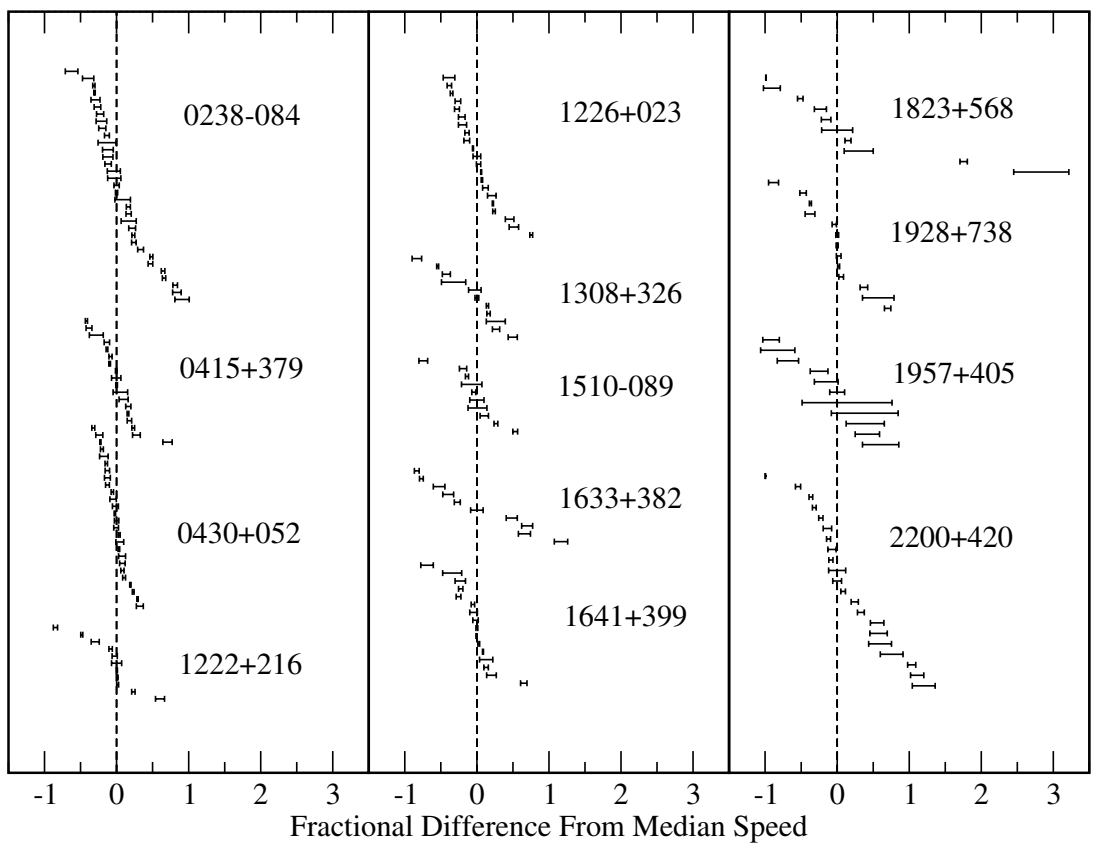

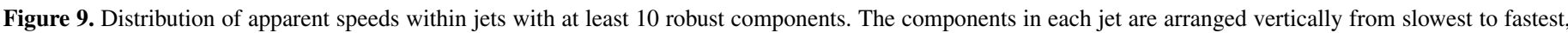
and the speeds are normalized with respect to the median speed of each jet. The fractional difference is defined as $\left(\mu-\mu_{\text {median }}\right) / \mu_{\text {median }}$.

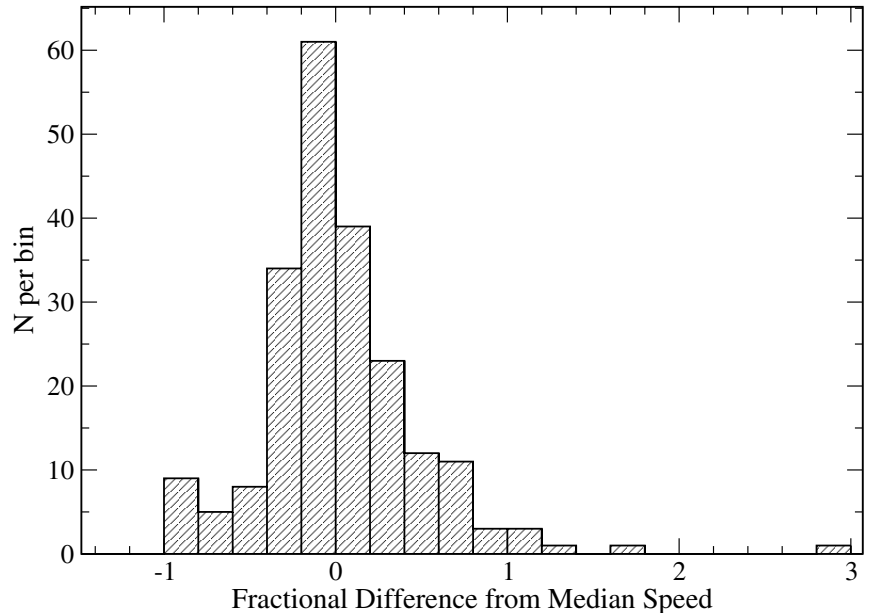

Figure 10. Overall normalized speed distribution within jets with at least 10 robust components. The fractional difference is defined as $\left(\mu-\mu_{\text {median }}\right) / \mu_{\text {median }}$

to eject features with apparent speeds that cluster about a speed that is characteristic to each individual jet.

A dispersion of apparent speeds within a jet might be expected in the context of our model presented in Section 5.2, in which features do not fill the entire jet cross section and emerge along a radial streamline within a conical outflow. Evidence of thin ribbon-like instabilities has been seen in the transversely resolved jet 3C 273 (Lobanov \& Zensus 2001) and the quasar S5 0836+710 (Perucho et al. 2012). The latter authors conclude that the ridgeline in VLBI images may correspond to a helically twisted pressure maximum within the jet, which slowly varies in position on decadal timescales. In the case of $3 \mathrm{C} 273$, polarimetric VLBA rotation measure maps made at different epochs by Zavala \& Taylor (2005), Asada et al. (2008), and Hovatta et al. (2012) indicate that different parts of the jet are being illuminated at different times. Similar behavior has also been reported in the broad-line radio galaxy 3C 120 by Gómez et al. (2011).

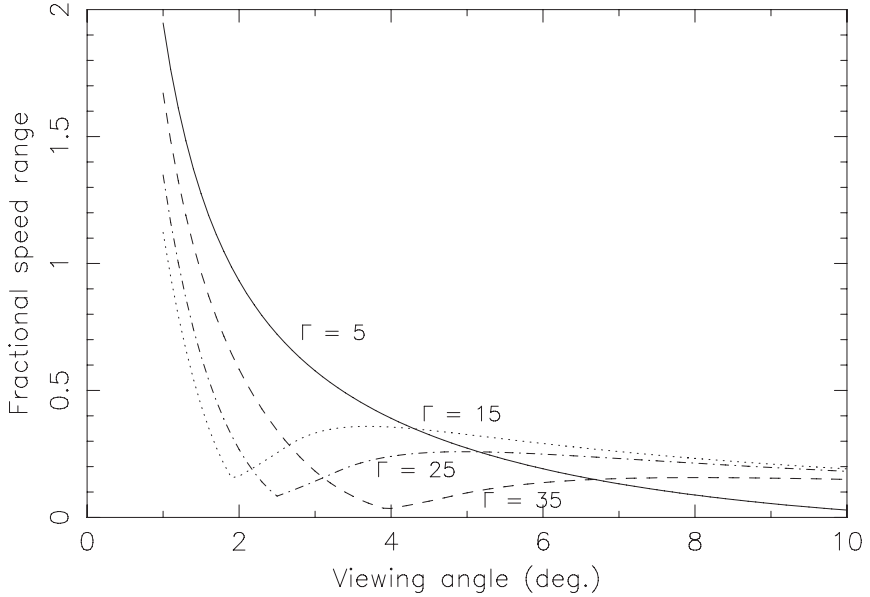

Figure 11. Expected range of apparent speed for features emitted within a conical jet of one degree half-angle, plotted against on-axis viewing angle. The $y$-axis values represent fractional difference from the on-axis apparent speed. The solid curve is for a jet with Lorentz factor 5, the dotted curve has Lorentz factor 15 , the dot-dashed curve Lorentz factor 25, and the dashed curve Lorentz factor 35 .

In our proposed scenario we would expect a range of apparent viewing angle roughly equal to the full opening angle of the ejection cone and a corresponding range in apparent speed. In Figure 11 we plot the range of expected apparent speed for conical jets with a $2^{\circ}$ full opening angle versus on-axis viewing angle. The $y$-axis values are normalized with respect to the on-axis apparent jet speed, i.e., a value of one indicates an apparent speed range as large as the on-axis apparent speed. The curves represent different bulk flow Lorentz factors and have minima at the critical angle $(1 / \Gamma)$ at which superluminal motion is maximum. Given the typically small fractional errors on our apparent speed measurements, the only cases where the predicted range approaches the FWHM of the data distribution $(\sim 1$; see Figure 10$)$ are for exceedingly aligned jets where the viewing angle is comparable to the jet opening angle. Since 


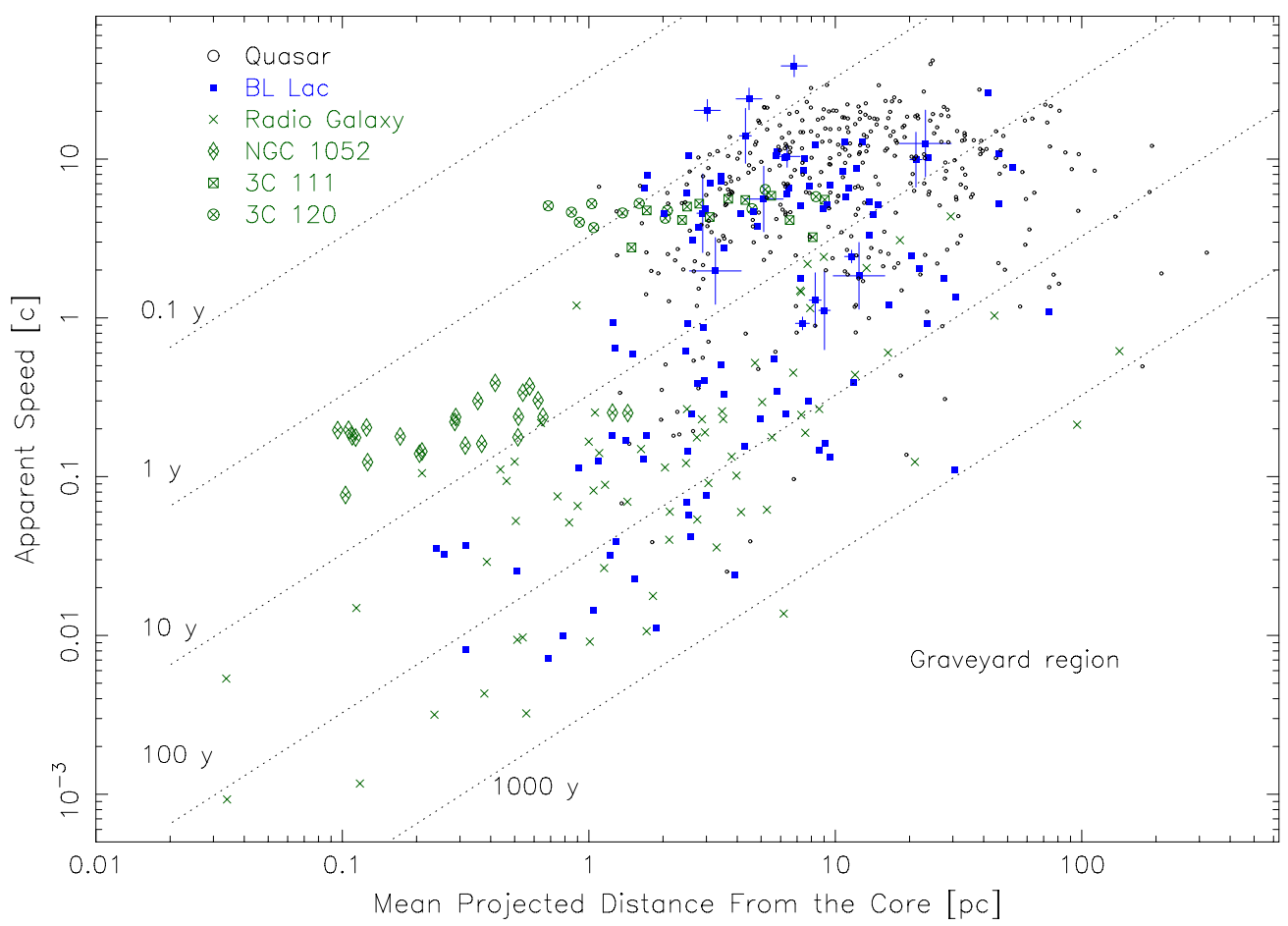

Figure 12. Apparent speed vs. mean projected distance from the core in parsecs for all robust jet components (excluding non-radial and inward components). The green crosses denote components in radio galaxies, the black circles quasars, and the blue squares BL Lac objects. The jet components of two broad-lined radio galaxies (3C 111 and 3C 120), as well as the gigahertz-peaked spectrum radio galaxy NGC 1052, are highlighted with distinct symbols. Error bars have been omitted for the purposes of clarity. For components in jets that do not have a spectroscopic redshift, we include range bars that correspond to their known redshift constraints (see Appendix). The dotted lines indicate lines of constant age for components that have moved steadily outward over the time period indicated. Newly emergent fast components rapidly evolve out of the top left region of the plot, while no components are found in the bottom right graveyard region since they will likely have faded well below the MOJAVE imaging sensitivity level.

(A color version of this figure is available in the online journal.)

the full opening angles of powerful blazar jets are typically less than $2^{\circ}$ (Jorstad et al. 2005; Pushkarev et al. 2009), and most blazar jets in a flux-density-limited sample will be viewed at approximately $1 / 2$ the critical superluminal motion angle (Vermeulen \& Cohen 1994), examples of jets viewed inside their opening angle should be rare. Such jets should be characterized by wide apparent opening angles and a large range in the sky position angles of ejected features (i.e., the outlier jets in Figure 5). We find no indications, however, that either of these two quantities is correlated with the range of apparent speed for the individual jets in our sample. We therefore conclude that the spread in apparent speed we see in individual jets cannot be wholly attributed to a spread in streamline viewing angle. The moving features must also have an intrinsic range of bulk Lorentz factor and/or pattern speed.

\subsection{Trends with Apparent Speed}

\subsubsection{Speed versus Distance Down the Jet}

In our analysis of MOJAVE and VLBA archive images from 1994 to 2007 (Papers VI and VII) we found that close to the base of the jet, features tended to show increasing rather than decreasing apparent speed. This suggests that AGN jet flows are still being organized on parsec-scales, a favored possible site for high-energy photon production. In order to test for this trend in our current data set, we have plotted the apparent speed of all robust components versus their mean projected distance from the core in Figure 12. We have omitted components with non-radial or inward motions and have included lines of constant age which assume steady radial motion over the indicated time period. Since the components in our sample have a wide range of ages, this would tend to suppress any artificial trend of higher mean distance for faster components. However, no components are found in the upper left corner of the plot, since these fast components will have higher mean core distance values and quickly evolve toward the right-hand side of the diagram. Also, sufficient time must pass to gather sufficient epochs for an apparent speed measurement in our survey. Components are also absent from the lower right "graveyard" region since these evolved components have likely undergone considerable adiabatic expansion and synchrotron energy losses, thereby dropping their flux densities below the threshold for which we can robustly measure their centroids and speeds. There is also a notable deficit of components above $\sim 0.4 c$ within $\sim 1 \mathrm{pc}$ of the core, which would be even more pronounced without the inclusion of the numerous components associated with the two-sided jets of the gigahertz-peaked spectrum galaxy NGC 1052. The two broad-line radio galaxies 3C 111 and 3C 120 also occupy a distinct region among the radio galaxy data plotted in Figure 12.

The overall distribution of the components in the plot indicates a positive correlation of speed with core distance for radio galaxies and BL Lac objects, even after partialling out redshift. This trend needs to be confirmed, however, using a larger, complete AGN sample that extends below $0.35 \mathrm{Jy}$. It is not possible to assess the existence of a trend for the quasars due to the observational selection effects described above. Because of the low space density of bright quasars at low redshift and the fixed angular resolution limit of our survey, there is likely an artificial deficit of quasar jet components in the region below $1 c$ and 


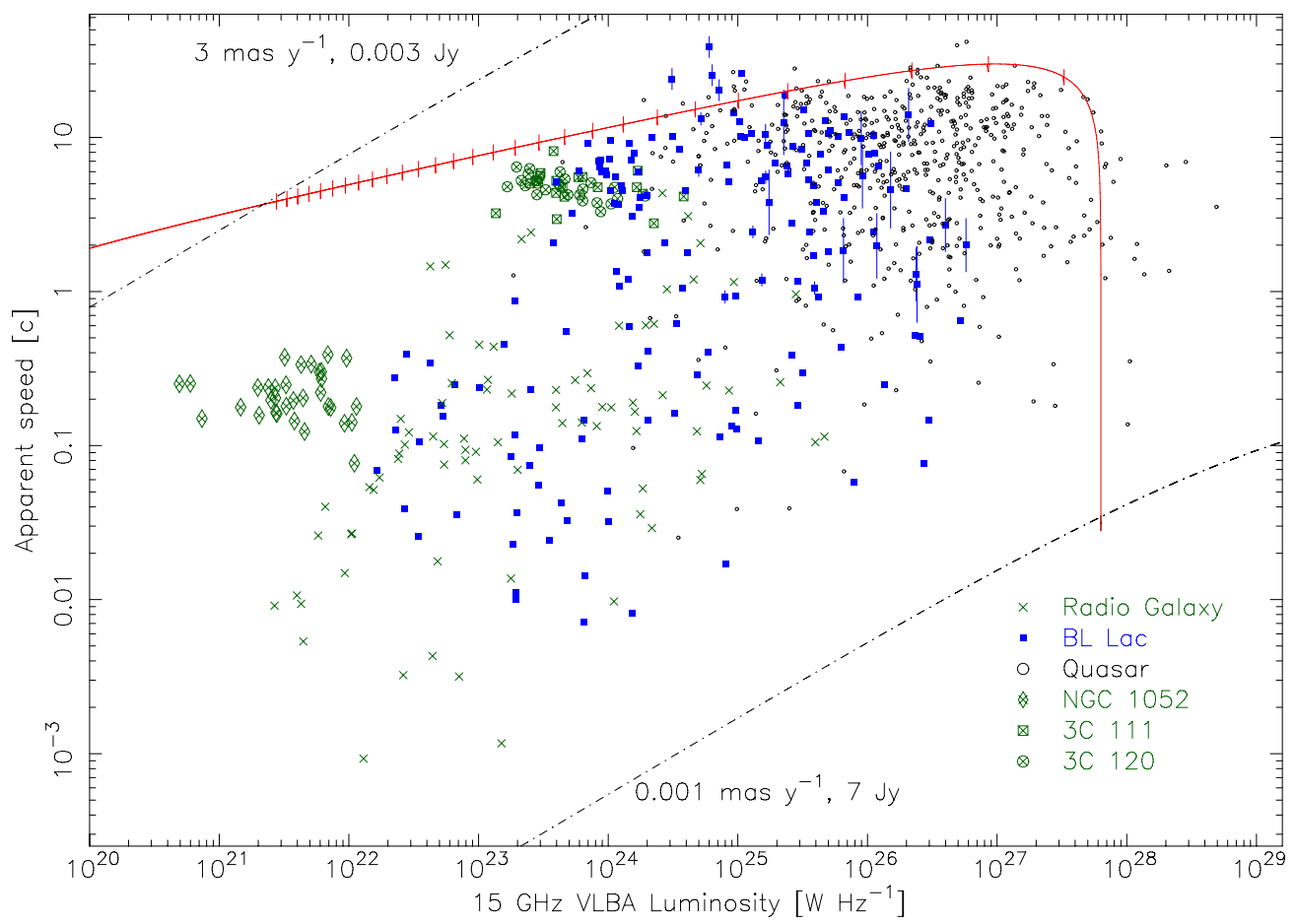

Figure 13. Apparent speed vs. $15 \mathrm{GHz}$ luminosity for all robust jet components (excluding inward components). The green crosses denote components in radio galaxies, the black circles quasars, and the blue squares BL Lac objects. Error bars have been omitted for purposes of clarity. For components in jets that do not have a spectroscopic redshift, we include vertical range bars that correspond to their known redshift constraints (see the Appendix). The red curve shows the locus of possible locations for a jet component with intrinsic luminosity $10^{23} \mathrm{~W} \mathrm{~Hz}^{-1}$, Doppler boost $\delta^{2.7}$, and a Lorentz factor $\Gamma=35$, viewed at different angles to the line of sight. The tick marks are drawn at $1^{\circ}$ intervals, ranging from a viewing angle of $30^{\circ}$ on the left to $1^{\circ}$ on the right. The dot-dashed lines correspond to observational limits; the regions in the top left and bottom right corners of the plot are not sampled by the survey.

(A color version of this figure is available in the online journal.)

within $\sim 1$ pc of the core, precisely where data are needed to test for a possible trend.

As we reported previously in Paper VI, the BL Lac jets have, on average, slower component speeds than the quasars. There are, however, many BL Lac jet components that overlap with the region populated by quasar jet components in Figure 12. Giommi et al. (2012) discuss the possibility of quasars masquerading as low equivalent line width BL Lac objects due to the swamping of their emission lines by highly Doppler boosted optical synchrotron emission. We note that one of the jet components with the fastest angular speed in our sample, ID $=16$ in the BL Lac object $0716+714$, is a distinct outlier in Figure 12, with recent constraints on its redshift by Danforth et al. (2013) implying an apparent speed of up to $43.6 c \pm 1.3 c$. This AGN is highly variable at all wavelengths (e.g., Wagner et al. 1996; Larionov et al. 2013) and has a Doppler factor of at least 20 (Rani et al. 2013), making it a strong candidate for a masquerading BL Lac object.

\subsubsection{Speed versus Luminosity}

A plot of apparent speed versus luminosity for the robust jet components (Figure 13) reveals a distinct deficit of fast components at low luminosities. The apparent upper envelope to this distribution has been discussed in several studies (Vermeulen 1995; Vermeulen \& Cohen 1994; Lister \& Marscher 1997; Cohen et al. 2007; Paper I; Paper VI) and roughly matches the locus of points for a jet of fixed intrinsic luminosity and bulk Lorentz factor, oriented at different angles to the line of sight (for $L>10^{23} \mathrm{~W} \mathrm{~Hz}^{-1}$ ). The parametric red curve in Figure 13 is drawn for a jet with $\Gamma=35, L_{\text {int }}=10^{23} \mathrm{~W} \mathrm{~Hz}^{-1}$, and Doppler boost $\delta^{2.7}$. This curve is representative only, since jets in the population have a range of Lorentz factors and intrinsic luminosities, and thus a family of such curves exist, as described by Cohen et al. (2007). The upper left and lower right regions of the plot (delimited by the dot-dashed lines) are not sampled by our survey. The deficit region located below the red curve and below $10^{23} \mathrm{~W} \mathrm{~Hz}^{-1}$ is partly due to the incompleteness and relatively high flux density cutoff of our low-luminosity sample. The overall fall-off in the upper edge of the distribution from $10^{26} \mathrm{~W} \mathrm{~Hz}^{-1}$ to $10^{23} \mathrm{~W} \mathrm{~Hz}^{-1}$ is not the result of survey bias or selection effects, however, and reflects both the existence of a maximum jet Lorentz factor in the parent population $(\sim 40)$ and an intrinsic correlation between flow speed and luminosity in AGN jets. The relatively sharp edge to the upper envelope in Figure 13 and the relatively unchanging distribution of apparent speed in luminosity bins above $10^{26} \mathrm{~W} \mathrm{~Hz}^{-1}$ imply that intrinsically powerful AGN jets have a wide range of Lorentz factors up to $\sim 40$, while intrinsically weak jets are only mildly relativistic.

\subsection{Accelerating Components}

Theoretical models of the acceleration and collimation of blazar jets indicate that the strong magnetic fields associated with the putative supermassive black hole/accretion disk system play a key role in the initial acceleration and collimation of the jet (e.g., Meier et al. 2001). While some models indicate that this process is largely complete with the conversion of Poynting flux to flow energy on sub-parsec scales (e.g., Sikora et al. 2005), there may still be significant magnetic (Vlahakis \& Königl 2004) or hydrodynamic acceleration that extends to parsec or decaparsec scales. 
In our previous analysis of MOJAVE data from 1994 to 2007 (Paper VII), we found that accelerated motions with respect to the mean apparent velocity vector $\boldsymbol{\beta}_{\text {obs }}$ were common, with significant parallel accelerations seen in roughly one-third of our sample, and significant perpendicular accelerations in about one-fifth of our sample. Due to the limited number of available epochs, we were only able to analyze 311 of the 526 moving components for possible accelerated motion. Using our new data spanning up to 2011 May 1, we have performed the same analysis on 547 of the 887 moving components in Table 5 which have at least 10 epochs. We summarize our overall acceleration results in Table 6 . Our incidence rates of parallel accelerations (28\%) and perpendicular accelerations (18\%) are consistent with those reported in Paper VII. Nearly $40 \%$ of the components analyzed show significant acceleration of either type. A substantial number of components showed no significant acceleration, but had non-radial motion vectors. If we assume that these components accelerated some time prior to our monitoring interval, the fraction of moving components showing accelerations rises to $70 \%$. This is in stark contrast to the kinematics of features in stellar (Herbig-Haro) jets, which are well described by ballistic models (e.g., Ellerbroek et al. 2013).

We note that our acceleration fits assume a simple two-dimensional parameterization in which the acceleration components in the R.A. and declination directions are constant over time. Impulsive or multiple changes in a component's velocity are therefore not well accommodated in our model. In Figure 14 we show some examples of component motions that are poorly represented by a simple constant acceleration fit. We discuss this issue, as well as the detailed acceleration properties of the full sample, in a future paper in this series.

\section{SUMMARY}

We present 1753 new multi-epoch VLBA images of 259 jets which are drawn from a complete radio-selected AGN sample above $1.5 \mathrm{Jy}$, a complete $\gamma$-ray-selected sample (Lister et al. 2011), and a representative low-luminosity radio jet sample. The latter is drawn from the VLBA Calibrator Survey and consists of 43 AGNs with $8 \mathrm{GHz}$ VLBA flux density greater than $0.35 \mathrm{Jy}$, $z \leqslant 0.3$ and $\mathrm{J} 2000$ declination $>-30^{\circ}$.

We have combined these new VLBA data with existing MOJAVE observations previously presented in Paper $\mathrm{V}$ and have analyzed the kinematics of 200 parsec-scale AGN jets. The data span 1994 August 31 to 2011 May 1 and were modeled with Gaussian components in the visibility plane. We obtained vector motion fits to 887 robust components which were positively cross-identified over at least five VLBA epochs. We also measured the acceleration properties of 557 components that had at least 10 VLBA epochs. Our main findings are as follows:

1. Nearly all of the 60 most heavily observed jets show significant changes in their innermost position angle $\left(20^{\circ}-150^{\circ}\right)$ over a 12-16 yr monitoring interval, with BL Lac jets showing smaller variations than quasars. The observed range corresponds to intrinsic (de-projected) variations of $\sim 0.5$ to $\sim 2^{\circ}$. Roughly half of the heavily observed jets display a trend of innermost jet position angle with time. In the case of 12 jets, there is some evidence of oscillatory behavior, but the fitted periods (5-12 yr) are too long compared to the length of the data set to firmly establish periodicity. These periods are very short compared to expected pre- cession timescales from warped accretion-disk-black-hole interactions associated with the Bardeen-Petterson effect (Natarajan \& Pringle 1998). Rather, we favor a conical jet model in which emerging features do not fill the entire cross section of the flow. What is typically visible in a single-epoch, limited dynamic range VLBA image as a series of bright features may actually be the lit-up portions of thin ribbon-like structures embedded within a broader conical outflow. These portions have been energized by a passing planar disturbance that originated in the VLBI core. As discussed by Perucho et al. (2012), the ribbon structures may arise from helical pressure maxima within the jet and slowly vary in position on decadal timescales.

2. We examined the distribution of speeds within 75 jets that had at least five robust components. Within a particular jet, the speeds of the features usually cluster around a characteristic value; however, the range of apparent speed among the components is comparable to the maximum speed measured within the jet. This is not due uniquely to unusually slow pattern speed components (defined as having a speed

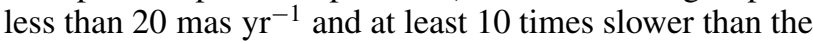
fastest component in the jet), which comprise only $4 \%$ of all the components studied and are less prevalent in quasar jets. It is also too large to be solely due to differences in ejection angle. We conclude that the dispersion is at least partially due to an intrinsic distribution of bulk Lorentz factor and/or pattern speed.

3. Apparent inward motions are rare, with only $2 \%$ of the components having apparent velocity vectors greater than $90^{\circ}$ from the outward jet direction. They occur more frequently in BL Lac jets. All of the detected inward motions are slow $\left(\lesssim 0.1\right.$ mas $\left.^{-1}\right)$, and nearly all occur within 1 mas of the core component.

4. We confirm a previously established upper envelope to the distribution of speed versus beamed luminosity for moving jet components. Below $10^{26} \mathrm{~W} \mathrm{~Hz}^{-1}$ there is a fall-off in maximum speed with decreasing $15 \mathrm{GHz}$ radio luminosity. The relatively sharp edge to the upper envelope and the relatively unchanging distribution of apparent speed in luminosity bins above $10^{26} \mathrm{~W} \mathrm{~Hz}^{-1}$ imply that intrinsically powerful AGN jets have a wide range of Lorentz factors up to $\sim 40$, while intrinsically weak jets are only mildly relativistic.

5. We find a trend of increasing apparent speed with distance down the jet for radio galaxies and BL Lac objects. The existence of a trend could not be evaluated for quasars due to unavoidable observational selection biases, as described in Section 5.4.1.

6. Accelerations are very common among the moving jet components. Of the 739 components with statistically significant $(\geqslant 3 \sigma)$ speeds, $38 \%$ exhibited significant non-radial motion, implying non-ballistic trajectories. We analyzed 547 components with at least 10 epochs and found $28 \%$ to have significant accelerations parallel to the velocity vector and $18 \%$ to have significant perpendicular accelerations. Nearly $40 \%$ showed significant acceleration of either type, and an additional $30 \%$ had non-radial motion vectors.

The MOJAVE program continues to gather VLBA data on the AGN jets in the samples presented here. Future papers in this series will discuss their polarization evolution, detailed acceleration characteristics, and connections between jet kinematics and other blazar properties such as $\gamma$-ray emission, flux variability, and synchrotron peak frequency. 

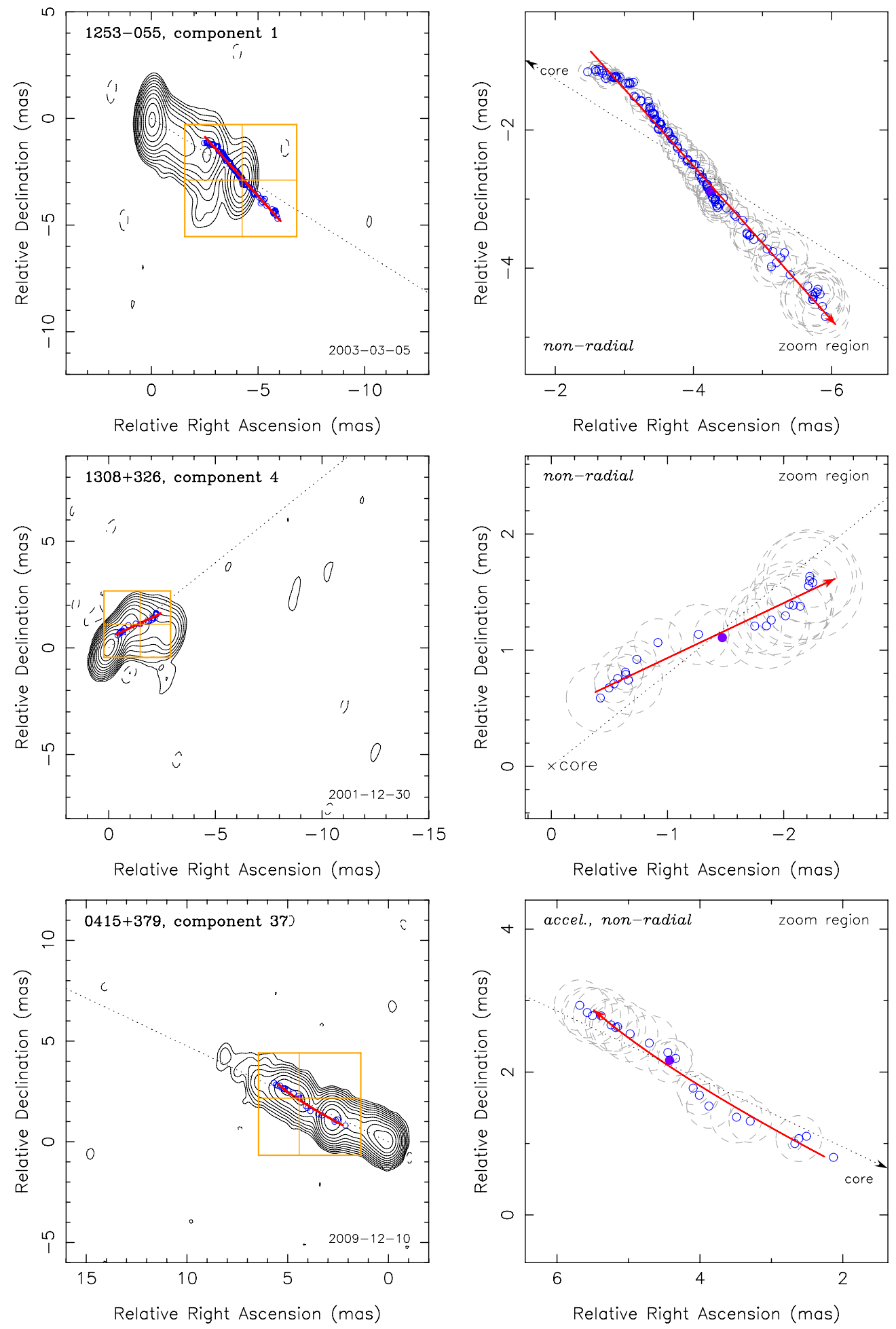

Figure 14. Vector motion fits and sky position plots of several robust jet components that show impulsive or multiple accelerations that are poorly fit by a simple constant acceleration model.

(A color version of this figure is available in the online journal.)

The MOJAVE project was supported under NASA-Fermi grants NNX08AV67G and 11-Fermi11-0019. E.R. was partially supported by the Spanish MINECO projects AYA200913036-C02-02 and AYA2012-38491-C02-01 and by the Generalitat Valenciana project PROMETEO/2009/104, as well as by the COST MP0905 action "Black Holes in a Violent Universe." M.F.A. and H.D.A. were supported by NASA-Fermi
GI grants NNX09AU16G, NNX10AP16G, and NNX11AO13G and NSF grant AST-0607523. Y.Y.K. was supported by the Russian Foundation for Basic Research (projects 12-02-33101, 13-02-12103), Research Program OFN-17 of the Division of Physics, Russian Academy of Sciences, and the Dynasty Foundation. A.B.P. was supported by the "Non-stationary processes in the Universe" Program of the Presidium of the Russian 
Academy of Sciences. D.C.H. was supported by NSF grant NSF AST-0707693. The National Radio Astronomy Observatory is a facility of the National Science Foundation operated under cooperative agreement by Associated Universities, Inc. This work made use of the Swinburne University of Technology software correlator (Deller et al. 2011), developed as part of the Australian Major National Research Facilities Programme and operated under license. The areaproportional Venn diagram was produced using the eulerAPE package.

\section{APPENDIX}

\section{NOTES ON INDIVIDUAL SOURCES}

0048-097. No distinct features could be reliably tracked in this very compact jet.

$0111+021$. The second and third closest components to the core in this nearby BL Lac object $(z=0.047)$ have significantly

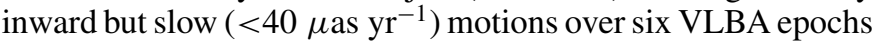
spanning $5 \mathrm{yr}$.

$0219+428$ (3C 66A). No reliable spectroscopic redshift exists for this BL Lac object. Furniss et al. (2013) have set limits of $0.3347 \leqslant z \leqslant 0.41$ based on intergalactic absorption features.

$0235+164$. The jet structure was too compact at $15 \mathrm{GHz}$ to reliably measure any robust components.

0238-084 (NGC 1052). Multi-frequency VLBA observations of this two-sided jet by Vermeulen et al. (2003) and Kadler et al. (2004) indicate that the core feature is obscured at $15 \mathrm{GHz}$ by strong free-free absorption associated with a circumnuclear torus. In order to obtain the component positions at each epoch, the position of a virtual core was found using a least-squares minimization method, as described by Vermeulen et al. (2003). The core component entries in Table 4 for this source are therefore left blank.

$0300+470$. No reliable redshift exists for this BL Lac object. Shaw et al. (2013) derive a statistical upper limit of $z<1.63$ based on the absence of intergalactic absorption features. With the addition of a new epoch in 2008, a component (ID = 2) first identified in Paper VI in this BL Lac jet now has a statistically significant inward motion.

$0430+289$. No reliable redshift exists for this BL Lac object. Shaw et al. (2013) derive a statistical upper limit of $z<1.66$ based on the absence of intergalactic absorption features, and Meisner \& Romani (2010) obtain $z>0.48$ based on optical non-detection of the host galaxy.

0506+056. This AGN is a Fermi LAT-detected, highsynchrotron peaked BL Lac object with unknown redshift. Meisner \& Romani (2010) obtain $z>0.38$ based on optical non-detection of the host galaxy, and Rau et al. (2012) set an upper limit $z<1.24$ based on the photometric redshift technique. A weak component on the western edge of this jet $(\mathrm{ID}=3)$ shows a statistically significant inward motion of

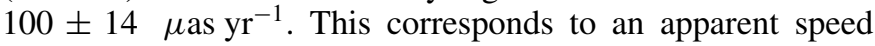
between $2.3 c$ and $6.1 c$, given the redshift limits.

$0716+332$. There were no components strong enough or sufficiently isolated to be considered robust in this jet.

$0716+714$. A spectroscopic redshift has yet to be obtained for this BL Lac object. Nilsson et al. (2008) published a value of $0.31 \pm 0.08$ based on a host galaxy magnitude estimate, while Danforth et al. (2013) have constrained the redshift to the range $0.2315<z<0.322$ using intervening absorption systems. We note that if the redshift is at the upper limit of this range, $0716+714$ becomes one of the fastest jets in our sample
$(43.6 c \pm 1.3 c)$. For all redshift values in the possible range, the jet is a distinct outlier in the $\beta_{\text {app }}$ versus luminosity plot (Figure 13).

0727-115. We did not identify any components in this jet as sufficiently robust to track over time.

$0814+425$. No reliable spectroscopic redshift exists for this BL Lac object. Sbarufatti et al. (2005a) found $z>0.75$ based on a lower limit to the host galaxy magnitude, and Shaw et al. (2013) derive a statistical upper limit of $z<2.47$ based on the absence of intergalactic absorption features. The innermost component of this jet showed significant inward motion between 1995 and 2006. We did not previously consider this component to be robust in Paper VI due to confusion arising from an emerging new component in 2007 (now identified as ID =6).

0946+006. There was too much positional scatter in the brightest downstream jet component (ID $=2$ ) to consider it as robust over the short $2 \mathrm{yr}$ long VLBA coverage of this AGN.

$0954+556(4 C+55.17)$. This unusual quasar is largely resolved by the VLBA at $15 \mathrm{GHz}$, yet has strong and variable $\gamma$-ray emission. McConville et al. (2011) have suggested that it may be a young radio source. We were not able to identify any robust components in this jet.

1011+496. This TeV-detected BL Lac object (1ES 1011+496; Albert et al. 2007) has a jet component moving at $1.8 c \pm 0.4 c$, making it a rare example of a superluminal high-synchrotron peaked blazar.

1124-186. The jet structure was too compact at $15 \mathrm{GHz}$ to reliably measure any robust components.

$1228+126$ (M87). We confirm the slow speeds we measured in Paper VI for this nearby radio galaxy. The closest component to the core in the main jet (ID =6) has a very slow pattern

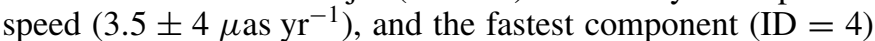
has significant non-radial motion at $0.026 c \pm 0.003 c$. These speeds are significantly slower than those measured in the HST1 feature $(0.6 c \pm 0.3 c$; Chang et al. 2010), located more than $80 \mathrm{pc}$ farther down the jet. Ly et al. (2007) found speeds of $0.25 c-0.4 c$ in the region $2-4$ mas downstream from the core, based on five VLBI epochs at 22 and $43 \mathrm{GHz}$ obtained between 1999 and 2004.

$1324+224$. The jet structure was too compact at $15 \mathrm{GHz}$ to reliably measure any robust components.

$1424+240$. No reliable spectroscopic redshift exists for this BL Lac object. Although Meisner \& Romani (2010) obtained $z=0.23$ based on its host galaxy magnitude, Shaw et al. (2013) subsequently set a firm lower limit of $z>0.6035$ based on intergalactic absorption features.

$1458+718$. We confirm the apparent inward motion reported in Paper VI of two components in a complex emission region located $\sim 25$ mas south of the core in this compact steep-spectrum quasar. With our new data we have found one additional component in this complex (ID =2) that is also moving inward, in a non-radial direction. The inward speeds of the three components range from $\sim 1.4 c$ to $4.6 c$.

$1509+054$. The radio structure of this AGN consists of three bright components. We identify the radio core as the middle component, based on the spectral index map of T. Hovatta et al. (in preparation).

$1637+826$. This nearby Seyfert 2 radio galaxy (also known as NGC 6251, at $z=0.024$ ) contains five outward-moving components with $\geqslant 3 \sigma$ speeds, all of which are below $0.15 c$. The innermost component (ID $=8$ ), however, has a small but significant inward motion of $50 \pm 10 \mu \mathrm{as} \mathrm{yr}^{-1}$ $(0.08 c \pm 0.015 c)$. 
$1739+522$. The jet structure was too compact at $15 \mathrm{GHz}$ to reliably measure any robust components.

1741-038. The jet structure was too compact at $15 \mathrm{GHz}$ to reliably measure any robust components.

$1823+568$. This BL Lac jet at $z=0.664$ has a very fast component speed: $26.2 c \pm 2.6 c$, as compared with the next fastest BL Lac component $(15.1 c \pm 0.4 c$ for $0851+202)$. It has been classified as a quasar by Véron-Cetty \& Véron (2006) and as a powerful FR II jet by Murphy et al. (1993) based on its extended radio emission. It therefore may be an intermediate BL Lac/quasar type object.

1921-293. The low declination and north-south orientation of this jet made it impossible to robustly track any of its bright features over time.

1958-179. The jet structure was too compact at $15 \mathrm{GHz}$ to reliably measure any robust components.

$1959+650$. The jet structure was too weak and compact at $15 \mathrm{GHz}$ to reliably measure any robust components.

$2005+403$. In Paper VI we reported a possible inward motion of a component very close to the core (ID $=6$ ); however, our new data indicate that there is too much complex sub-structure in this region to reliably determine robust component positions.

$2021+614$. We find the two outermost components (ID $=1$ and 2) to have inward motions, albeit with very slow speeds (7.2 and $15.2 \mu \mathrm{as} \mathrm{yr}^{-1}$, respectively). The fitted motion vector for component 1 is non-radial. The core component location in this jet remains uncertain (see Paper VI).

$2023+335$. The radio structure in this low galactic latitude quasar $(-2.4)$ is too strongly affected by interstellar scattering to permit the tracking of robust jet features (Pushkarev et al. 2013).

2200+420 (BL Lacertae). The innermost component (ID = 7) of this jet showed very little motion and an uncertain vector motion direction in our Paper VI analysis. With the addition of many new epochs since 2007, we now find the component to have a very slow but significant motion of $3.8 \pm 0.6 \mu{\mathrm{as} \mathrm{yr}^{-1}}^{-1}$ $(0.017 c \pm 0.003 c)$.

$2201+171$. Our previous analysis in Paper VI suggested inward motion for component 3 , but subsequent data revealed that its fitted position after 2007 was likely affected by a new rapidly outward-moving component (ID $=6$ ). As a result, we have categorized component 3 as non-robust.

2230+114 (CTA 102). In Paper VI we reported a single jet component at $\sim 6$ mas (ID $=4$ ) as having significant inward motion, which is re-affirmed with the most recent data.

2247-283. All of the fitted jet features in this source were weaker than $100 \mathrm{mJy}$, and none could be reliably tracked over the five available epochs.

$2351+456$. After reporting an inward-moving component $(\mathrm{ID}=2)$ in Paper VI, we subsequently re-performed the model fits to all epochs, including the new data, and now find no statistically significant motion for this component $(\mu=40 \pm 14$ $\mu$ as $\left.\mathrm{yr}^{-1}\right)$.

\section{REFERENCES}

Abazajian, K., Adelman-McCarthy, J. K., Agüeros, M. A., et al. 2004, AJ, 128,502

Abazajian, K., Adelman-McCarthy, J. K., Agüeros, M. A., et al. 2005, AJ, 129,1755

Abdo, A. A., Ackermann, M., Ajello, M., et al. 2010, ApJ, 715, 429

Ackermann, M., Ajello, M., Allafort, A., et al. 2011, ApJ, 743, 171

Adelman-McCarthy, J. K., Agüeros, M. A., Allam, S. S, et al. 2006, ApJS, 162,38
Adelman-McCarthy, J. K., Agüeros, M. A., Allam, S. S., et al. 2008, ApJS, 175,297

Agudo, I. 2009, in ASP Conf. Ser. 402, Approaching Micro-Arcsecond Resolution with VSOP-2: Astrophysics and Technologies, ed. Y. Hagiwara, E. Fomalont, M. Tsuboi, \& M. Yasuhiro (San Francisco, CA: ASP), 330 Agudo, I., Bach, U., Krichbaum, T. P., et al. 2007, A\&A, 476, L17

Agudo, I., Marscher, A. P., Jorstad, S. G., et al. 2012, ApJ, 747, 63

Akiyama, M., Ueda, Y., Ohta, K., Takahashi, T., \& Yamada, T. 2003, ApJS, 148, 275

Albert, J., Anderhub, H., Antoranz, P., et al. 2007, ApJL, 667, L21

Aldcroft, T. L., Bechtold, J., \& Elvis, M. 1994, ApJS, 93, 1

Asada, K., Inoue, M., Kameno, S., \& Nagai, H. 2008, ApJ, 675, 79

Bach, U., Krichbaum, T. P., Ros, E., et al. 2005, A\&A, 433, 815

Baker, J. C., Hunstead, R. W., Kapahi, V. K., \& Subrahmanya, C. R. 1999, ApJS, 122,29

Bartel, N., Shapiro, I. I., Huchra, J. P., \& Kuhr, H. 1984, ApJ, 279, 112

Beasley, A. J., Gordon, D., Peck, A. B., et al. 2002, ApJS, 141, 13

Best, P. N., Peacock, J. A., Brookes, M. H., et al. 2003, MNRAS, 346, 1021

Boisse, P., \& Bergeron, J. 1988, A\&A, 192, 1

Boksenberg, A., Briggs, S. A., Carswell, R. F., Schmidt, M., \& Walsh, D. 1976, MNRAS, 177, 43P

Browne, I. W. A., Savage, A., \& Bolton, J. G. 1975, MNRAS, 173, 87P

Burbidge, E. M. 1970, ApJL, 160, L33

Cara, M., \& Lister, M. L. 2008, ApJ, 674, 111

Carangelo, N., Falomo, R., Kotilainen, J., Treves, A., \& Ulrich, M.-H. 2003, A\&A, 412, 651

Chang, C. S., Ros, E., Kovalev, Y. Y., \& Lister, M. L. 2010, A\&A, 515, A38

Chavushyan, V., Mujica, R., Gorshkov, A. G., et al. 2001, ARep, 45, 79

Cohen, M. H., Lister, M. L., Homan, D. C., et al. 2007, ApJ, 658, 232

Cohen, R. D., Smith, H. E., Junkkarinen, V. T., \& Burbidge, E. M. 1987, ApJ, 318,577

Danforth, C. W., Nalewajko, K., France, K., \& Keeney, B. A. 2013, ApJ, 764,57

de Grijp, M. H. K., Keel, W. C., Miley, G. K., Goudfrooij, P., \& Lub, J. 1992, A\&AS, 96, 389

Deller, A. T., Brisken, W. F., Phillips, C. J., et al. 2011, PASP, 123, 275

Denicoló, G., Terlevich, R., Terlevich, E., et al. 2005, MNRAS, 356, 1440

di Serego-Alighieri, S., Danziger, I. J., Morganti, R., \& Tadhunter, C. N. 1994, MNRAS, 269, 998

Drinkwater, M. J., Webster, R. L., Francis, P. J., et al. 1997, MNRAS, 284, 85

Duncan, G. C., \& Hughes, P. A. 1994, ApJL, 436, L119

Eckart, A., Witzel, A., Biermann, P., et al. 1986, A\&A, 168, 17

Ellerbroek, L. E., Podio, L., Kaper, L., et al. 2013, A\&A, 551, A5

Ellison, S. L., Yan, L., Hook, I. M., et al. 2001, A\&A, 379, 393

Eracleous, M., \& Halpern, J. P. 1994, ApJS, 90, 1

Eracleous, M., \& Halpern, J. P. 2004, ApJS, 150, 181

Falomo, R., Scarpa, R., \& Bersanelli, M. 1994, ApJS, 93, 125

Fomalont, E. B. 1999, in ASP Conf. Ser. 180, Synthesis Imaging in Radio Astronomy II, ed. G. B. Taylor, C. L. Carilli, \& R. A. Perley (San Francisco, CA: ASP), 301

Fomalont, E. B., Petrov, L., MacMillan, D. S., Gordon, D., \& Ma, C. 2003, AJ, 126,2562

Fricke, K. J., Kollatschny, W., \& Witzel, A. 1983, A\&A, 117, 60

Furniss, A., Fumagalli, M., Danforth, C., Williams, D. A., \& Prochaska, J. X. 2013, ApJ, 766, 35

Gelderman, R., \& Whittle, M. 1994, ApJS, 91, 491

Giommi, P., Padovani, P., Polenta, G., et al. 2012, MNRAS, 420, 2899

Giovannini, G., Cotton, W. D., Feretti, L., Lara, L., \& Venturi, T. 2001, ApJ, 552,508

Glikman, E., Helfand, D. J., White, R. L., et al. 2007, ApJ, 667, 673

Gómez, J. L., Roca-Sogorb, M., Agudo, I., Marscher, A. P., \& Jorstad, S. G. 2011, ApJ, 733, 11

Halpern, J. P., \& Eracleous, M. 1997, IAUC, 6639, 2

Halpern, J. P., Eracleous, M., \& Mattox, J. R. 2003, AJ, 125, 572

Hardee, P. E. 2011, in IAU Symp. 275, Jets at All Scales, ed. G. E. Romero, R. A. Sunyaev, \& T. Belloni (Cambridge: Cambridge Univ. Press), 41

Healey, S. E., Romani, R. W., Cotter, G., et al. 2008, ApJS, 175, 97

Heidt, J., Tröller, M., Nilsson, K., et al. 2004, A\&A, 418, 813

Henstock, D. R., Browne, I. W. A., Wilkinson, P. N., \& McMahon, R. G. 1997, MNRAS, 290, 380

Hewett, P. C., Foltz, C. B., \& Chaffee, F. H. 1995, AJ, 109, 1498

Ho, L. C., \& Kim, M. 2009, ApJS, 184, 398

Homan, D. C., Kadler, M., Kellermann, K. I., et al. 2009, ApJ, 706, 1253

Homan, D. C., Ojha, R., Wardle, J. F. C., et al. 2002, ApJ, 568, 99

Hook, I. M., McMahon, R. G., Irwin, M. J., \& Hazard, C. 1996, MNRAS, 282,1274

Hovatta, T., Lister, M. L., Aller, M. F., et al. 2012, AJ, 144, 105 
Huchra, J. P., Vogeley, M. S., \& Geller, M. J. 1999, ApJS, 121, 287

Hunstead, R. W., Murdoch, H. S., \& Shobbrook, R. R. 1978, MNRAS, 185, 149

Hunter, S. D., Bertsch, D. L., Dingus, B. L., et al. 1993, ApJ, 409, 134

Jackson, N., \& Browne, I. W. A. 1991, MNRAS, 250, 414

Jauncey, D. L., Batty, M. J., Wright, A. E., Peterson, B. A., \& Savage, A. 1984, ApJ, 286, 498

Jones, D. H., Read, M. A., Saunders, W., et al. 2009, MNRAS, 399, 683

Jones, D. H., Saunders, W., Read, M., \& Colless, M. 2005, PASA, 22, 277

Jorstad, S. G., Marscher, A. P., Lister, M. L., et al. 2004, AJ, 127, 3115

Jorstad, S. G., Marscher, A. P., Lister, M. L., et al. 2005, AJ, 130, 1418

Junkkarinen, V. 1984, PASP, 96, 539

Kadler, M., Ros, E., Lobanov, A. P., Falcke, H., \& Zensus, J. A. 2004, A\&A, 426, 481

Kellermann, K. I., Lister, M. L., Homan, D. C., et al. 2004, ApJ, 609, 539

Kellermann, K. I., Vermeulen, R. C., Zensus, J. A., \& Cohen, M. H. 1998, AJ, 115,1295

Kovalev, Y. Y., Petrov, L., Fomalont, E. B., \& Gordon, D. 2007, AJ, 133, 1236

Landoni, M., Falomo, R., Treves, A., et al. 2012, A\&A, 543, A116

Larionov, V. M., Jorstad, S. G., Marscher, A. P., et al. 2013, ApJ, 768, 40

Lawrence, C. R., Pearson, T. J., Readhead, A. C. S., \& Unwin, S. C. 1986, AJ, 91, 494

Lawrence, C. R., Zucker, J. R., Readhead, A. C. S., et al. 1996, ApJS, 107, 541

Lister, M. L., Aller, H. D., Aller, M. F., et al. 2009a, AJ, 137, 3718

Lister, M. L., Aller, M., Aller, H., et al. 2011, ApJ, 742, 27

Lister, M. L., Cohen, M. H., Homan, D. C., et al. 2009b, AJ, 138, 1874

Lister, M. L., \& Homan, D. C. 2005, AJ, 130, 1389

Lister, M. L., \& Marscher, A. P. 1997, ApJ, 476, 572

Lobanov, A. P., \& Roland, J. 2005, A\&A, 431, 831

Lobanov, A. P., \& Zensus, J. A. 2001, Sci, 294, 128

Lomb, N. R. 1976, Ap\&SS, 39, 447

Ly, C., Walker, R. C., \& Junor, W. 2007, ApJ, 660, 200

Lynds, C. R. 1967, ApJ, 147, 837

Marcha, M. J. M., Browne, I. W. A., Impey, C. D., \& Smith, P. S. 1996, MNRAS, 281,425

Marti-Vidal, I., Marcaide, J. M., Alberdi, A., \& Brunthaler, A. 2013, arXiv:1301.4782

Marziani, P., Sulentic, J. W., Dultzin-Hacyan, D., Calvani, M., \& Moles, M. 1996, ApJS, 104, 37

Marziani, P., Sulentic, J. W., Zamanov, R., et al. 2003, ApJS, 145, 199

McConville, W., Ostorero, L., Moderski, R., et al. 2011, ApJ, 738, 148

McIntosh, D. H., Rieke, M. J., Rix, H.-W., Foltz, C. B., \& Weymann, R. J. 1999, ApJ, 514, 40

Meier, D. L., Koide, S., \& Uchida, Y. 2001, Sci, 291, 84

Meisner, A. M., \& Romani, R. W. 2010, ApJ, 712, 14

Michel, A., \& Huchra, J. 1988, PASP, 100, 1423

Murphy, D. W., Browne, I. W. A., \& Perley, R. A. 1993, MNRAS, 264, 298

Natarajan, P., \& Pringle, J. E. 1998, ApJL, 506, L97

Nilsson, K., Pursimo, T., Sillanpää, A., Takalo, L. O., \& Lindfors, E. 2008, A\&A, 487, L29

Nilsson, K., Pursimo, T., Villforth, C., et al. 2012, A\&A, 547, 1

Orr, M. J. L., \& Browne, I. W. A. 1982, MNRAS, 200, 1067

Osmer, P. S., Porter, A. C., \& Green, R. F. 1994, ApJ, 436, 678

Owen, F. N., Ledlow, M. J., Morrison, G. E., \& Hill, J. M. 1997, ApJL, 488, L15

Perlman, E. S., Padovani, P., Giommi, P., et al. 1998, AJ, 115, 1253

Perlman, E. S., Stocke, J. T., Schachter, J. F., et al. 1996, ApJS, 104, 251

Perucho, M., Kovalev, Y. Y., Lobanov, A. P., Hardee, P. E., \& Agudo, I. 2012, ApJ, 749, 55

Peterson, B. A., Wright, A. E., Jauncey, D. L., \& Condon, J. J. 1979, ApJ, 232, 400

Petrov, L., Kovalev, Y. Y., Fomalont, E., \& Gordon, D. 2005, AJ, 129, 1163

Petrov, L., Kovalev, Y. Y., Fomalont, E. B., \& Gordon, D. 2006, AJ, 131, 1872

Petrov, L., Kovalev, Y. Y., Fomalont, E. B., \& Gordon, D. 2008, AJ, 136, 580

Pietsch, W., Bischoff, K., Boller, T., et al. 1998, A\&A, 333, 48

Piner, B. G., Pant, N., \& Edwards, P. G. 2010, ApJ, 723, 1150

Pita, S., Goldoni, P., Boisson, C., et al. 2012, in AIP Conf. Proc. 1505, High Energy Gamma-Ray Astronomy: 5th International Meeting on High Energy Gamma-Ray Astronomy, ed. F. A. Aharonian, W. Hofmann, \& F. M. Rieger (Melville, NY: AIP), 566

Pushkarev, A. B., Hovatta, T., Kovalev, Y. Y., et al. 2012, A\&A, 545, A113

Pushkarev, A. B., Kovalev, Y. Y., Lister, M. L., \& Savolainen, T. 2009, A\&A, 507, L33

Pushkarev, A. B., Kovalev, Y. Y., Lister, M. L., et al. 2013, A\&A, 555, 80

Rani, B., Krichbaum, T. P., Fuhrmann, L., et al. 2013, A\&A, 552, A11
Rau, A., Schady, P., Greiner, J., et al. 2012, A\&A, 538, A26

Rector, T. A., \& Stocke, J. T. 2001, AJ, 122, 565

Rosen, A., Hughes, P. A., Duncan, G. C., \& Hardee, P. E. 1999, ApJ, 516, 729 Sargent, W. L. W. 1970, ApJ, 160, 405

Savage, A., Browne, I. W. A., \& Bolton, J. G. 1976, MNRAS, 177, 77P

Savolainen, T., Wiik, K., Valtaoja, E., \& Tornikoski, M. 2006, A\&A, 446, 71

Sbarufatti, B., Treves, A., \& Falomo, R. 2005a, ApJ, 635, 173

Sbarufatti, B., Treves, A., Falomo, R., et al. 2005b, AJ, 129, 559

Scargle, J. D. 1982, ApJ, 263, 835

Schachter, J. F., Stocke, J. T., Perlman, E., et al. 1993, ApJ, 412, 541

Schmidt, M. 1977, ApJ, 217, 358

Schneider, D. P., Hall, P. B., Richards, G. T., et al. 2005, AJ, 130, 367

Schneider, D. P., Hall, P. B., Richards, G. T., et al. 2007, AJ, 134, 102

Schneider, D. P., Richards, G. T., Fan, X., et al. 2002, AJ, 123, 567

Schneider, D. P., Richards, G. T., Hall, P. B., et al. 2010, AJ, 139, 2360

Schramm, K.-J., Borgeest, U., Kuehl, D., et al. 1994, A\&AS, 106, 349

Searle, L., \& Bolton, J. G. 1968, ApJL, 154, L101

Shaw, M. S., Romani, R. W., Cotter, G., et al. 2012, ApJ, 748, 49

Shaw, M. S., Romani, R. W., Cotter, G., et al. 2013, ApJ, 764, 135

Shepherd, M. C. 1997, in ASP Conf. Ser. 125, Astronomical Data Analysis Software and Systems VI, ed. G. Hunt \& H. E. Payne (San Francisco, CA: ASP), 77

Sikora, M., Begelman, M. C., Madejski, G. M., \& Lasota, J.-P. 2005, ApJ, 625,72

Small, T. A., Sargent, W. L. W., \& Steidel, C. C. 1997, AJ, 114, 2254

Smith, H. E., Burbidge, E. M., Baldwin, J. A., et al. 1977, ApJ, 215, 427

Smith, R. J., Lucey, J. R., Hudson, M. J., Schlegel, D. J., \& Davies, R. L. 2000, MNRAS, 313, 469

Sowards-Emmerd, D., Romani, R. W., \& Michelson, P. F. 2003, ApJ, 590, 109

Sowards-Emmerd, D., Romani, R. W., Michelson, P. F., Healey, S. E., \& Nolan, P. L. 2005, ApJ, 626, 95

Spinrad, H., Marr, J., Aguilar, L., \& Djorgovski, S. 1985, PASP, 97, 932

Steidel, C. C., \& Sargent, W. L. W. 1991, ApJ, 382, 433

Stickel, M., Fried, J. W., \& Kuehr, H. 1988, A\&A, 191, L16

Stickel, M., Fried, J. W., \& Kuehr, H. 1989, A\&AS, 80, 103

Stickel, M., Fried, J. W., \& Kuehr, H. 1993a, A\&AS, 98, 393

Stickel, M., \& Kuehr, H. 1993, A\&AS, 100, 395

Stickel, M., Kuehr, H., \& Fried, J. W. 1993b, A\&AS, 97, 483

Stickel, M., Meisenheimer, K., \& Kuehr, H. 1994, A\&AS, 105, 211

Stirling, A. M., Cawthorne, T. V., Stevens, J. A., et al. 2003, MNRAS, 341,405

Stocke, J. T., Wurtz, R., Wang, Q., Elston, R., \& Jannuzi, B. T. 1992, ApJL, 400, L17

Strauss, M. A., Huchra, J. P., Davis, M., et al. 1992, ApJS, 83, 29

Strittmatter, P. A., Carswell, R. F., Gilbert, G., \& Burbidge, E. M. 1974, ApJ, 190, 509

Tadhunter, C. N., Morganti, R., di Serego-Alighieri, S., Fosbury, R. A. E., \& Danziger, I. J. 1993, MNRAS, 263, 999

Thompson, D. J., Djorgovski, S., \& de Carvalho, R. 1990, PASP, 102, 1235

Thompson, D. J., Djorgovski, S., Vigotti, M., \& Grueff, G. 1992, ApJS, 81, 1

Ulrich, M.-H., Kinman, T. D., Lynds, C. R., Rieke, G. H., \& Ekers, R. D. 1975, ApJ, 198, 261

Valtonen, M. J., \& Wiik, K. 2012, MNRAS, 421, 1861

Vermeulen, R. C. 1995, PNAS, 92, 11385

Vermeulen, R. C., \& Cohen, M. H. 1994, ApJ, 430, 467

Vermeulen, R. C., Ogle, P. M., Tran, H. D., et al. 1995, ApJL, 452, L5

Vermeulen, R. C., Ros, E., Kellermann, K. I., et al. 2003, A\&A, 401, 113

Véron-Cetty, M.-P., \& Véron, P. 2006, A\&A, 455, 773

Vlahakis, N., \& Königl, A. 2004, ApJ, 605, 656

Wagner, S. J., Witzel, A., Heidt, J., et al. 1996, AJ, 111, 2187

Walsh, D., Beckers, J. M., Carswell, R. F., \& Weymann, R. J. 1984, MNRAS, 211,105

Walsh, D., \& Carswell, R. F. 1982, MNRAS, 200, 191

Wegner, G., Bernardi, M., Willmer, C. N. A., et al. 2003, AJ, 126, 2268

White, G. L., Jauncey, D. L., Wright, A. E., et al. 1988, ApJ, 327, 561

White, R. L., Becker, R. H., Gregg, M. D., et al. 2000, ApJS, 126, 133

Wilkes, B. J. 1986, MNRAS, 218, 331

Wills, D., \& Lynds, R. 1978, ApJS, 36, 317

Wills, D., \& Wills, B. J. 1974, ApJ, 190, 271

Wills, D., \& Wills, B. J. 1976, ApJS, 31, 143

Wright, A. E., Ables, J. G., \& Allen, D. A. 1983, MNRAS, 205, 793

Xu, W., Lawrence, C. R., Readhead, A. C. S., \& Pearson, T. J. 1994, AJ, 108,395

Zavala, R. T., \& Taylor, G. B. 2005, ApJL, 626, L73

Zensus, J. A., Ros, E., Kellermann, K. I., et al. 2002, AJ, 124, 662 